Revue des patrimoines

25 | 2014

Le patrimoine de la Grande Guerre

\title{
Printemps 1915 dans les tranchées : les lettres et le carnet de croquis de Pierre Vergez
}

\section{Brigitte Fromaget}

\section{Q OpenEdition}

1 Journals

\section{Electronic version}

URL: http://journals.openedition.org/insitu/11495

DOI: 10.4000/insitu. 11495

ISSN: $1630-7305$

\section{Publisher}

Ministère de la Culture

\section{Electronic reference}

Brigitte Fromaget, «Printemps 1915 dans les tranchées : les lettres et le carnet de croquis de Pierre Vergez », In Situ [Online], 25 | 2014, Online since 29 December 2014, connection on 25 June 2020. URL : http://journals.openedition.org/insitu/11495 ; DOI : https://doi.org/10.4000/insitu.11495

This text was automatically generated on 25 June 2020 .

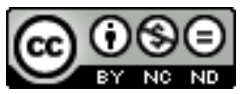

In Situ Revues des patrimoines est mis à disposition selon les termes de la licence Creative Commons Attribution - Pas d'Utilisation Commerciale - Pas de Modification 4.0 International. 


\title{
Printemps 1915 dans les tranchées : les lettres et le carnet de croquis de Pierre
} Vergez

\author{
Brigitte Fromaget
}

1 Mobilisé au moment où il commence à percer comme créateur d'affiches de spectacles parisiens, Pierre Vergez ne cesse de dessiner dans la boue des tranchées. Dans les lettres qu'il envoie à son père, il décrit avec humour et distanciation le quotidien des poilus, qu'il illustre de croquis pittoresques. Mais son carnet de dessins montre une réalité plus cruelle avec ses morts et ses villages en ruines (fig. $\mathbf{1}^{1}$ ). 


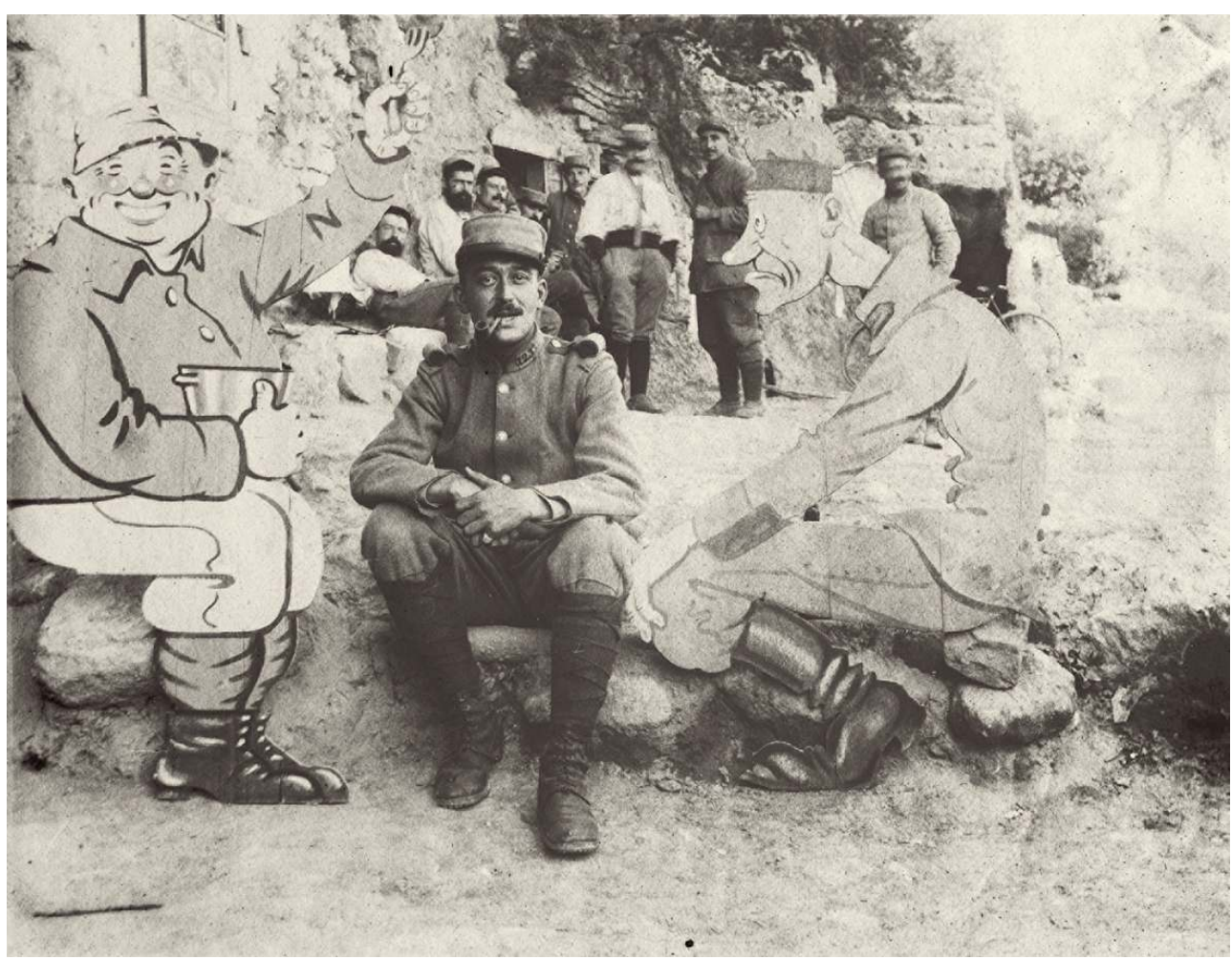

Pierre Vergez posant entre deux caricatures de soldats : à gauche le Français replet brandissant joyeusement sa fourchette, à droite l'Allemand famélique le regardant avec envie.

Repro. Barbe-Richaud, Pierre-Marie. (c) Droits réservés.

2 Pierre Vergez naît le 2 avril 1881 à Bordeaux. Sylvain, son père, quatrième enfant d'une famille d'agriculteurs des Hautes-Pyrénées, fait des études pour être fonctionnaire des impôts indirects. En 1880, il épouse Adèle Fleury, fille d'un professeur de littérature issu d'une vieille famille de Bagnères-de-Bigorre. Après avoir occupé des postes dans plusieurs villes, dont Bordeaux, où naissent ses enfants Pierre et Marguerite, Sylvain termine sa carrière à La Rochelle en 1912. Veuf depuis 1904, il s'installe alors à Aureilhan, près de Tarbes, avant de mourir en 1923, à quelques kilomètres de là, à Séméac, chez sa fille et son gendre, Gabriel Fromaget.

\section{De la vie parisienne à la vie des tranchées}

Bien que très attiré par le dessin et l'écriture, Pierre suit des études de droit pour faire plaisir à son père, et, en 1907, soutient une thèse sur la délinquance juvénile. Son doctorat en poche, il « monte » à Paris pour se consacrer à sa passion, le dessin et la peinture. Dans une lettre envoyée à sa grand-mère en 1910, il écrit :

Malgré la persuasion de beaucoup de gens que la vie d'artiste est une vie de tapage et de plaisirs, je mène la vie la plus calme qui soit, et bien moins mouvementée que celle de beaucoup de petits employés parisiens. Je vous aurai donc tout dit de moi quand vous saurez que mes affaires vont, sans hausses exagérées, mais aussi sans baisses affolantes, sans grand espoir pour l'avenir, sans regret pourtant d'une carrière qui me laisse du moins mon entière liberté. Je bricole à droite et à gauche, pour l'affiche, pour les journaux, pour les éditeurs, et même pour les fabricants de 
jouets. Les mois opulents compensent les mois de purée et les balthazars font oublier les dinettes où le pain tient un rôle par trop prépondérant.

4 Les années suivantes, il concentre son activité sur les affiches de théâtres et de spectacles parisiens qu'il signe "Verjez» avec un J, croquant de nombreux artistes de la Belle Époque. Dans les années 1909-1913, il illustre aussi des partitions de chansons de variétés avec des figures de femmes élégantes ou des croquis satiriques ${ }^{2}$. Il commence à être connu dans la capitale lorsqu'éclate la guerre de 1914-1918. Immédiatement mobilisé comme sergent d'infanterie, il est envoyé en première ligne et endure la guerre des tranchées, où il exerce ses talents en illustrant de croquis pittoresques, pris sur le vif, certaines lettres qu'il adresse à son entourage. Ayant survécu aux combats des fantassins et à la boue des tranchées, il a la malchance à quelques mois de sa démobilisation et comme tant d'autres poilus, de contracter une grippe infectieuse qui l'emporte le 2 avril 1919 au centre hospitalier de Zillisheim, près de Mulhouse ${ }^{3}$.

De sa correspondance avec son père à qui il écrivait deux fois par semaine environ, ne subsistent que dix-sept lettres dont seize illustrées, envoyées entre le 29 mars et le 11 juin 1915, les autres ont malheureusement disparu. Ont également été retrouvés un carnet de croquis et de dessins, à la mine ou à l'encre, rehaussés de crayons de couleur, ainsi que deux photographies et quelques documents épars: figures et caricatures de poilus dont un « cuistot » avec définition au dos, une feuille de chêne piquetée portant le mot ARGONNE et le portrait du général Hirschauer qui avait signé le droit au port de la fourragère aux couleurs du ruban de la Médaille militaire conféré à son régiment en 1917 (fig. 2). Le dernier témoignage, daté du 6 juin 1918, est un court message écrit à l'encre de Chine sur un fragment de toile : « sur ce débris d'une aile d'avion boche descendu près de nous ce matin je t'envoie mon affectueux souvenir ». 


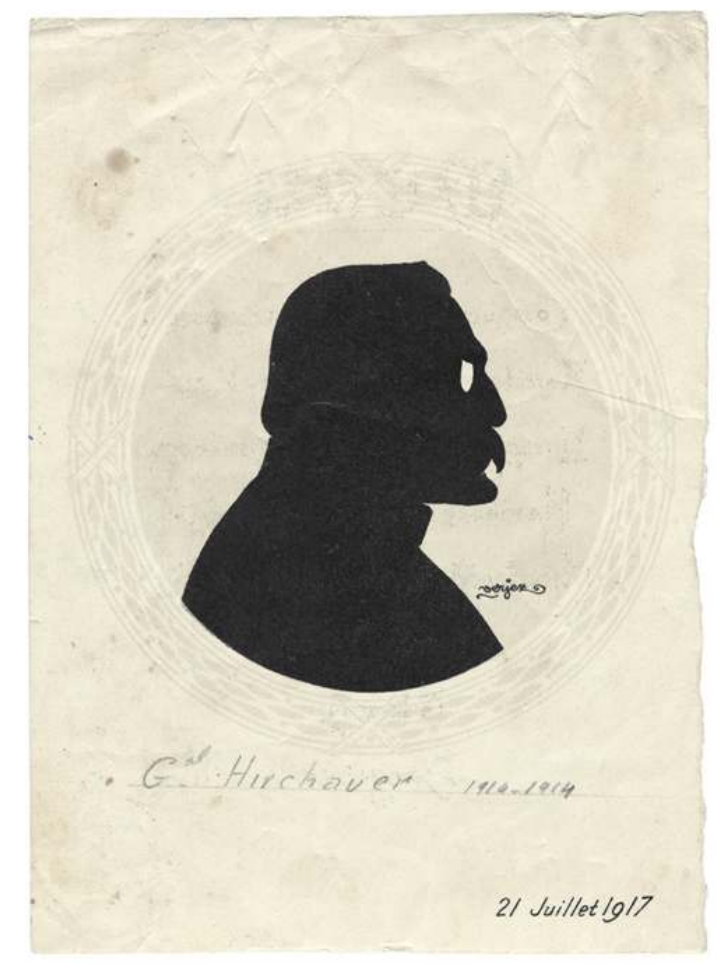

Portrait du général Hirschauer.

Repro. Barbe-Richaud, Pierre-Marie. (c) Droits réservés.

En août 1914, Pierre Vergez est incorporé dans le $123^{\mathrm{e}}$ régiment d'infanterie de La Rochelle $^{4}$, qui est transporté par chemin de fer à Barisey-la-Côte, en Meurthe-et-Moselle, puis dirigé vers la frontière belge. Après dix jours de retraite sans repos, les bataillons reprennent, le 6 septembre, l'offensive à Montceau-lès-Provins - ce qui vaudra au régiment une première citation au corps d'armée - et participent à la bataille de la Marne. Quelques jours plus tard, ils repoussent de fortes attaques dans le bois de BeauMarais, au sud de Craonne. En novembre, après de violents combats le $123^{\mathrm{e}} \mathrm{RI}$ prend la zone de Moussy-Verneuil-Beaulne et occupe les premières tranchées, fossés remplis de boue, sans abri ni boyau de communication. Le 12 janvier 1915, il relève un autre régiment dans le secteur Vendresse-et-Troyon mais, le 16, les Allemands font exploser une mine et s'emparent de l'entonnoir ainsi créé. La situation est rétablie par une contreattaque, et c'est le début de la longue guerre des mines du secteur de Troyon.

7 La guerre de mouvement avec ses terribles offensives a échoué. L'arrêt brutal des Allemands modifie la défense, les armées s'enterrent et se retranchent, commence alors la guerre de position avec une stratégie d'usure. Il s'agit moins d'avancer que de tenir le terrain et d'infliger de lourdes pertes à l'ennemi pour l'affaiblir. Désormais, la tranchée devient le cadre de la vie et de la mort du soldat. C'est de là que Pierre Vergez envoie à son père, entre le 29 mars et 11 juin 1915, des lettres où humour, dessins et caricatures estompent l'horreur de la réalité (fig. 3). 


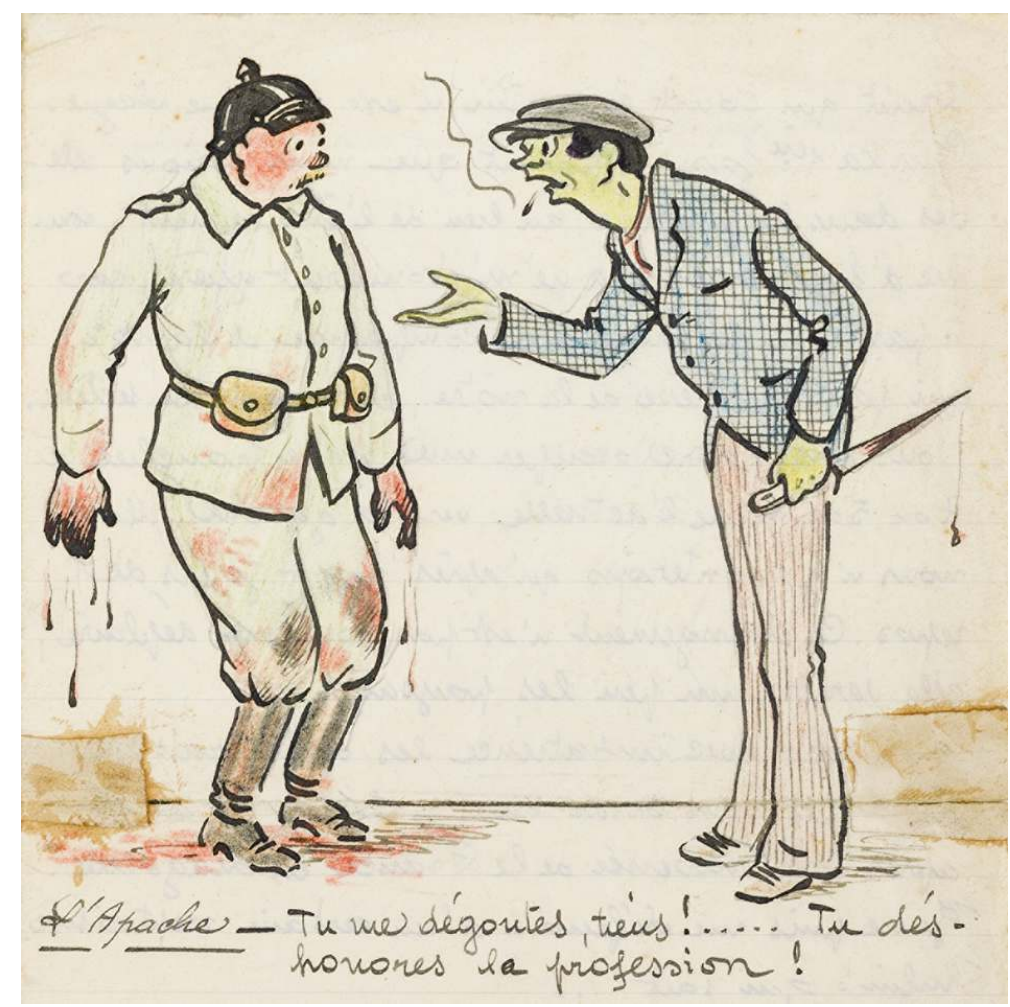

Rencontre improbable d'un soldat allemand et d'un Apache.

Repro. Barbe-Richaud, Pierre-Marie. (c) Droits réservés.

Il ne parle jamais directement des affrontements ni des tués, des blessés, des disparus qui se comptent déjà par milliers, pourtant il se trouve depuis le début des hostilités en plein cœur des combats. Il décrit et illustre la vie quotidienne dans les tranchées sans rien omettre, ni le froid, ni la pluie et la boue, ni le manque d'hygiène, ni les pilonnages d'artillerie mais il minimise tout et ne se plaint jamais. Pour rassurer son père, il fait en sorte de gommer l'horreur quotidienne des tranchées avec la mort toujours présente. Bien que deux fois moins âgé que lui, le fils de R. Kipling emploie la même tactique dans ses lettres : humour, anecdotes, préoccupations culinaires et vestimentaires qui peuvent apporter un peu de confort, mais aucune allusion à la peur ${ }^{5}$. Au contraire, quand Pierre évoque la mitraille et les pluies de projectiles qui survolent les tranchées, ses commentaires sont atténués par un "c'est amusant » ou "c'est rigolo », sans doute pour conjurer l'angoisse. Il prend moins de précautions lorsqu'il s'adresse à sa tante dont les deux fils sont sous les drapeaux: l'un, médecin major au Maroc, dans les troupes de Lyautey, sera tué en 1916 à Fez, l'autre, dans les tranchées, survivra à la guerre; leur mère en sait probablement plus que son beau-frère sur ce qui se passe dans les champs de bataille. Le 7 mai 1915, Pierre lui expose franchement son sentiment sur la suite du conflit :

J'ai l'impression que nous préparons seulement la vraie guerre, et que ce qui s'est passé jusqu'ici n'était qu'un acte premier d'une pièce qui en comporte sans doute plusieurs. Les hommes d'ici le pensent et sont prêts à attendre six mois aussi bien qu'à marcher demain. En est-il de même des populations des villes? Je me doute que beaucoup de gens doivent lire les communiqués, et hausser chaque matin les épaules en disant: «Peuh !... si c'est tout ce qu'ils ont fait hier, ce n'était vraiment 
pas la peine!» Á ceux-là, il faut dire qu'ils y viennent voir eux-mêmes; il faut arriver à les convaincre qu'une ligne d'un communiqué, la plus brève soit-elle, représente un effort terrible et beaucoup de chair déchirée ou refroidie ; et que tout est si bien organisé comme défense, d'un côté comme de l'autre, qu'une avance de $500 \mathrm{~m}$. peut être considérée comme une vraie victoire. J'ai peur que la patience s'émousse plus vite chez les civils que chez les soldats; cela serait énorme de conséquences, et c'est pourquoi je désire tant que ceux qui restent soient au moins aussi vaillants que nous dans la résignation et inébranlables dans la confiance.

\section{Organisation et aménagement des tranchées}

9 Les lignes ennemies sont séparées par un no man's land plus ou moins étendu variant entre quelques mètres et quelques kilomètres. Cette zone est soumise aux tirs d'artillerie des deux camps et, lors des assauts, les pertes sont extrêmement lourdes et font partie intégrante de cette guerre de positions. Les tranchées se répartissent sur des lignes parallèles pour créer plusieurs points de résistance successive ; elles communiquent entre elles par d'étroits boyaux. À l'arrière se trouvent les cantonnements de repos et les postes de commandement avec les services de ravitaillement et les infirmeries. Les bataillons passent de la première ligne aux secondes puis au lieu de repos. Mais cette organisation fait suite aux énormes travaux d'aménagement des tranchées que Pierre Vergez explique fort bien dès sa première lettre illustrée.

10 Tranchée, 29 mars 1915 :

Ce soir descente à Troyon (pays des rats) pour 4 jours de repos au cours desquels nous allons fabriquer les claies qui nous servent ici de planchers et de murs, et les trébuchets de fil de fer barbelé que l'on place devant nos tranchées de façon à établir une ligne presque infranchissable pour les boches (fig. 4). 


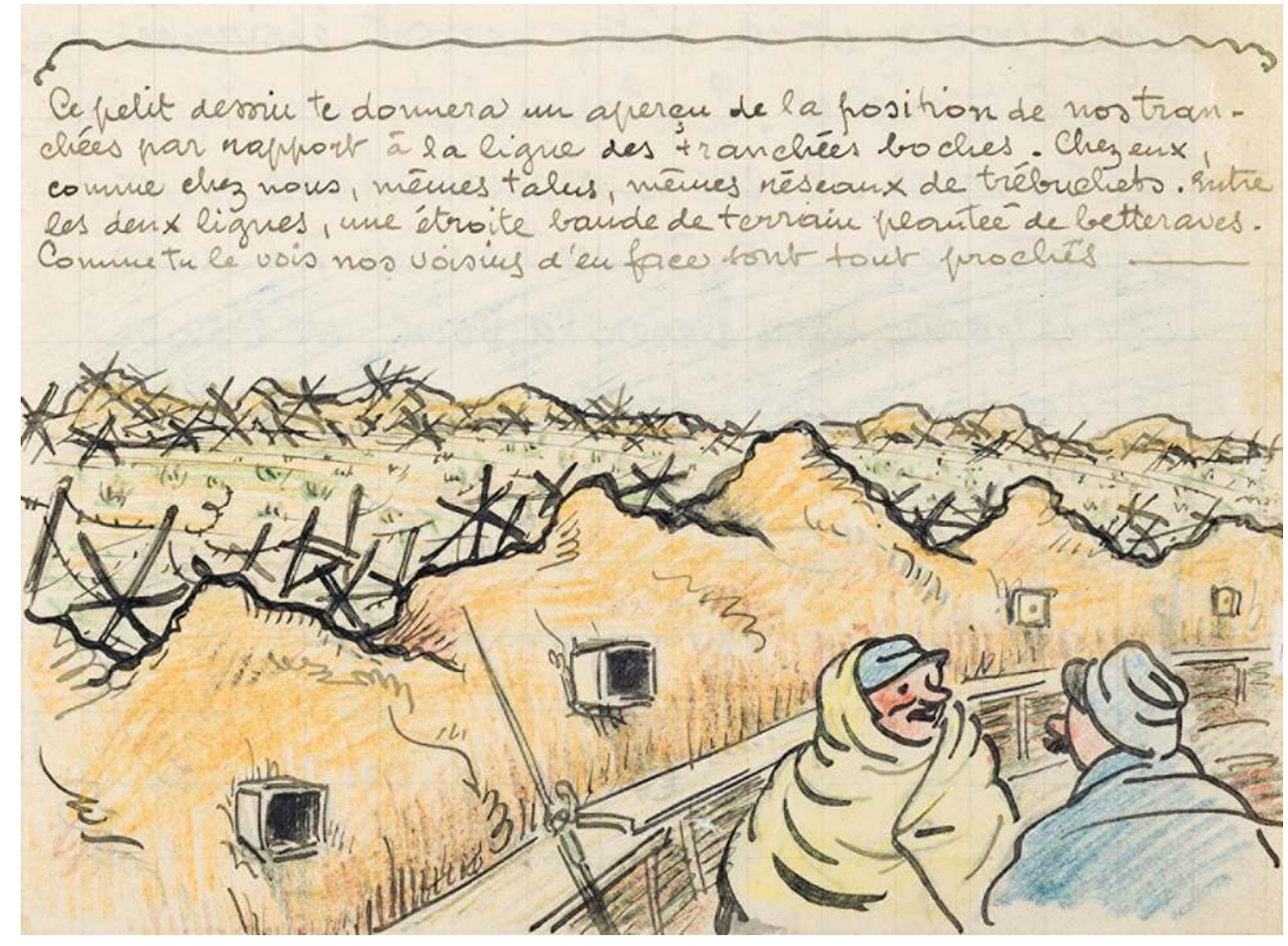

Disposition et proximité des tranchées ennemies.

Repro. Barbe-Richaud, Pierre-Marie. @ Droits réservés.

Troyon, $1^{\text {er }}$ avril :

Je pars tout à l'heure, bien que nous soyons censés être au repos, avec une équipe de terrassiers pour travailler à l'établissement d'une tranchée de $3^{\mathrm{e}}$ ligne qui est en bonne voie. J'y passerai probablement l'après-midi et souhaite qu'il fasse le même temps qu'hier. Hier matin la campagne était toute blanche de neige. Certaines prairies où se creusent de nombreux trous de marmites boches, prenaient sous la neige l'aspect de paysages lunaires avec leurs montagnes en forme de cuvettes. Puis le soleil a percé les nuages et tout le jour il a fait une bonne chaleur, par moments presque accablante. Si cela se maintient aujourd'hui, la corvée de terrassement sera un plaisir.

Le 6 avril :

Hier, profitant d'une accalmie, j'ai été visiter la tranchée de la $9^{\mathrm{e}} \mathrm{Cie}$, un peu à gauche de la nôtre. Le clou de cette tranchée est un point, dénommé Point $\mathrm{U}$, où la ligne boche et la nôtre ne sont éloignées que de 6 à 7 mètres. Les trébuchets sont presque à se toucher. C'est invraisemblable, et pourtant cela est. D'une tranchée à l'autre l'on se parle sans élever la voix, et l'on se jette des journaux, des cigarettes, des étuis de cartouches.

Le 12 avril Pierre envoie à son père une carte d'état-major :

... nos tranchées sont approximativement au crayon bleu qui coupe le chemin des Dames. L'emplacement de celles du $12^{\mathrm{e}}$ est à peu près à la ligne de crayon bleu, sous Hurtebise. C'est d'ailleurs la même tranchée qui se poursuit probablement sans discontinuer.

Le 17 avril, il commente un de ses dessins de la tranchée :

À gauche, au premier plan le tombeau sommaire d'un soldat inconnu. Plusieurs sont tombés par-là lors des derniers combats de janvier. Sont-ils allemands ou français, 
on n'en sait en général rien. Leur présence a été révélée seulement pendant les travaux d'élargissement des tranchées. Plusieurs sont si proches de la paroi qu'il faut continuellement arroser au chlore à cause des infiltrations et des émanations. ( fig. 5)

Figure 5

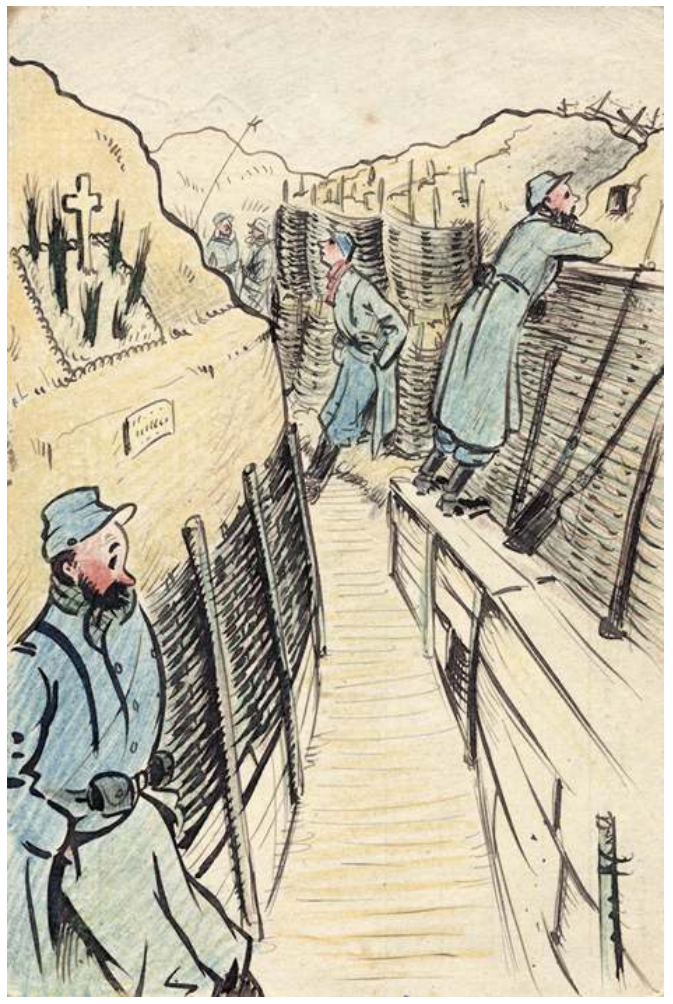

Tombe d'un soldat inconnu dans le talus de la tranchée. Repro. Barbe-Richaud, Pierre-Marie. (c) Droits réservés.

\section{Concert et boules puantes}

Cette guerre n'a plus rien à voir avec les précédentes, ce que n'a pas compris Rudyard Kipling qui fait du « tourisme » dans les tranchées. « Des tranchées-témoins » lui réplique son fils après avoir lu son récit, pas celles qu'un bataillon français creuse en une nuit. L'invraisemblable conseil de protéger les tranchées des bombes avec du grillage à lapins fait également réagir le fils ${ }^{6}$. La guerre est devenue une guerre de matériel, une guerre industrielle.

La guerre des tranchées nécessite de nouvelles armes adaptées à ce type de défense inédite : les grenades à main, armes légères capables d'atteindre les soldats terrés et les abris fragiles, l'artillerie de tranchée qui tire à faible distance sous des angles très ouverts, et divers types d'explosifs dont le « crapouillot » chez les Français. Derrière cet armement de première ligne sont installées des batteries à trajectoire courbe pour atteindre les retranchements, et enfin les grosses pièces d'artillerie lourdes. Il ne faut pas non plus négliger l'entrée de l'aviation dans la guerre. Cependant toutes ces nouveautés n'excluent pas le corps à corps à l'arme blanche. 
Voici comment Pierre Vergez évoque ce type d'arsenal à Troyon le $1^{\text {er }}$ avril : «... c'est vraiment rigolo de se doucher dans le bruit de la fusillade qui crépite à $300 \mathrm{~m}$. en avant et des marmites qui éclatent à $200 \mathrm{~m}$. en arrière ». Trois jours plus tard dans la tranchée, il en dit plus, toujours sur un ton humoristique qu'il faut savoir décoder :

Heureusement, tous les jours nous avons l'apéritif-concert. Cela consiste à entendre passer sur nos têtes, à diverses reprises, quelque chose comme 300 obus de 75 en 5 minutes. À l'aide des périscopes, on peut voir à très courte distance, voler en l'air de la terre, des pieux, des linges divers. C'est très amusant. Les canons de 75 étant placés derrière les ondulations de nos lignes arrière, ils sont obligés de tirer très bas, pour atteindre les tranchées boches de $1^{\text {ère }}$ lignes à peine distantes des nôtres de 25 à $30 \mathrm{~m}$. en moyenne. Ce tir demande une extraordinaire précision, que seules nos pièces possèdent. Tous ces obus passent à peine à 1 ou 2 mètres au-dessus de nos propres talus, avec des sifflements très musicaux qui constituent le chant de la partition. L'éclatement de tous ces colis en face de nous, forment l'accompagnement de l'orchestre, dans les notes basses. Tout cela ne va pas très bien en mesure ; c'est néanmoins de la bonne musique moderne.

Il ajoute le 12 avril, « toujours le petit train-train des balles et des obus».

\section{Le 3 mai 1915 :}

Les boches ont recommencé à bombarder Moulins pendant notre séjour aux tranchées. Cela n'était pas arrivé depuis longtemps, si ces saligauds continuent, l'on n'y sera pas plus en sécurité qu'en première ligne et peut-être même moins encore. C'est un peu la faute de nos chefs. C'est très bien de faire des marches et un peu d'exercice. Malheureusement tout cela se fait en plein air, et les aviateurs boches n'ont pas les lorgnettes dans leurs poches. Il est vrai que notre artillerie leur répond en bombardant Cerny qui est un des lieux de rassemblement de nos adversaires directs. Cerny! Quand tu verras ce nom dans les communiqués, cela voudra dire que nous aurons marché, car c'est le patelin qui fait pendant à Moulins de l'autre côté des tranchées.

Nous voilà à Moulins depuis hier soir, pour quatre jours. Pendant notre absence la localité a été bombardée sérieusement. Quelques blessés, à peine trois morts. Les chevaux seuls ont souffert sérieusement, car plusieurs écuries ont été touchées. Chevaux de troupe surtout... Aujourd'hui encore, quelques obus sont tombés, mais dès le premier coup les hommes savent dans quelle cave ils doivent se réfugier; aussi le risque de pertes est-il réduit à son minimum.

21 Tranchées, 24 mai :

Notre nouveau secteur (environ $400 \mathrm{~m}$. à gauche de l'ancien) diffère peu de l'ancien. Les abris sont cependant plus renforcés, le secteur moins vaste. La distance des boches, peut-être un peu plus grande, mais ce n'est pas un avantage, car nous sommes mieux placés pour recevoir torpilles et crapouillauds. Nous en avons eu avant-hier une abondante distribution. Ce sont de bien antipathiques engins. Leurs grosses torpilles contiennent 50 kilos d'explosifs (sorte de mélinite), c'est dire les déplacements d'air et de matériaux dont elles sont capables. Heureusement le départ a un bruit caractéristique, et comme leur marche est très lente, on les suit de l'œil en l'air et il est relativement facile de s'en garer.

22 Autres nouveautés dans l'armement, l'utilisation des gaz toxiques par les Allemands depuis avril. Pierre y fait allusion dans une lettre écrite dans les tranchées le 16 mai :

Nous avons fait ces jours derniers une répétition générale de tactique à suivre au cas où nos saligauds de voisins nous enverraient des boules puantes. C'est malheureux d'avoir à faire la guerre dans de pareilles conditions. Enfin! Nous n'aurons que plus de mérite à en venir à bout, et ce ne sont pas leurs procédés nouveaux qui leur attireront les amitiés dont ils auraient tant besoin. 
Tranchées, $1^{\mathrm{er}}$ juin :

Nous venons de toucher divers accessoires dont le dessin ci-contre te donnera la forme et la destination. Nous portons toujours la calotte (casque d'acier pour protection contre la densité du feu et les éclats d'obus) dans les tranchées. Les lunettes et le sachet ne nous serviraient que pour marcher à l'attaque ou pour recevoir nos adversaires. En plus, contre le vitriol, il faudrait nous enduire de vaseline ; mais nous n'en avons pas encore touché. Si tu peux trouver de la vaseline en petit tube, comme mes peintures à l'huile, envoie m'en. Idem un crayon chinois $\mathrm{n}^{\circ} 2$ (c'est la marque des crayons que tu achetais jadis en paquet)... Ici, calme relatif, avec crises violentes mais brèves (fig. 6).

Figure 6

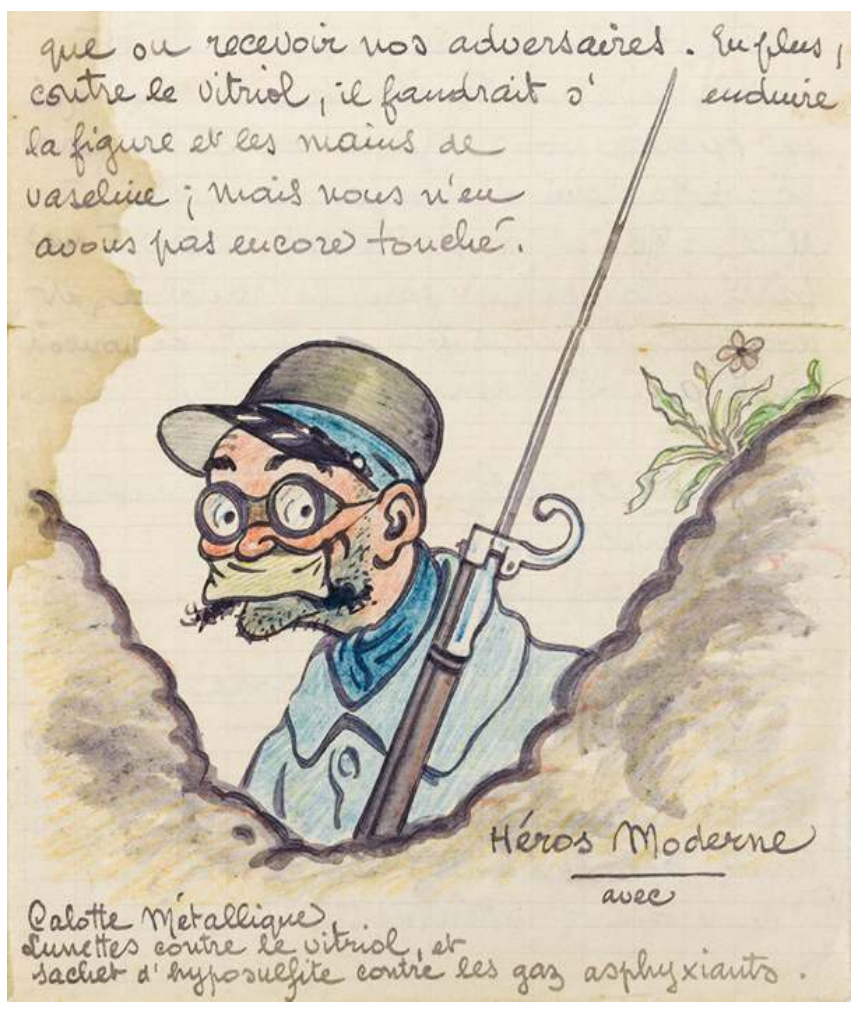

Poilu prêt à affronter les gaz toxiques.

Repro. Barbe-Richaud, Pierre-Marie. (c) Droits réservés.

Le 7 juin: "L'on s'occupe toujours de nous prémunir contre les armes puantes ou corrosives de nos adversaires et nous avons reçu ce matin quelques cagoules en étoffe avec œillères en mica. C'est très rigolo ». Les premiers obus à gaz seront lancés dans le secteur en novembre 1915.

\section{Santé, bains-douches et infirmeries}

La boue, les immondices, les rats, les poux et insectes de toutes sortes, l'air pollué par les gaz chimiques et la fumée, les morts ou les blessés agonisant à proximité des tranchées, rendent la vie particulièrement difficile. C'est pourquoi l'une des principales préoccupations du poilu est de rassurer les siens sur sa santé.

Tranchée, 29 mars 1915 : 
Je ne suis pas malade le moins du monde, seuls le sac et les côtes me fatiguent et m'essoufflent... Ton idée que j'avais pu recevoir des soins de dames de la Croix-

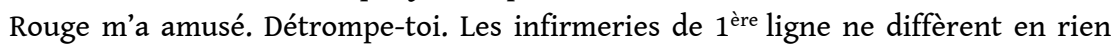
des cantonnements ordinaires. Mêmes litières de paille où les hommes s'entassent pêle-mêle, soit dans des pièces, soit dans des caves. Un drapeau à la porte et c'est tout; quelques majors qui circulent et une pharmacie tout à fait embryonnaire. Quelques brancardiers ou infirmiers, mais pas la moindre hygiène. Troyon, $1^{\mathrm{er}}$ avril :

À Troyon (300 m. des tranchées) voilà que l'on installe, comme on l'a déjà fait à Moulins, un établissement de bains-douches. C'est très rudimentaire, comme tu le penses : un tonneau, perché à $2 \mathrm{~m}$. en l'air, avec quelques petits trous dans le fond. Comme cuve, une grande lessiveuse trouvée dans le patelin. Et la farce est jouée. Ça ne va pas toujours très vite, et l'eau n'est pas toujours très chaude, cependant l'hygiène des poilus y trouve son compte (fig. 7).

Figure 7

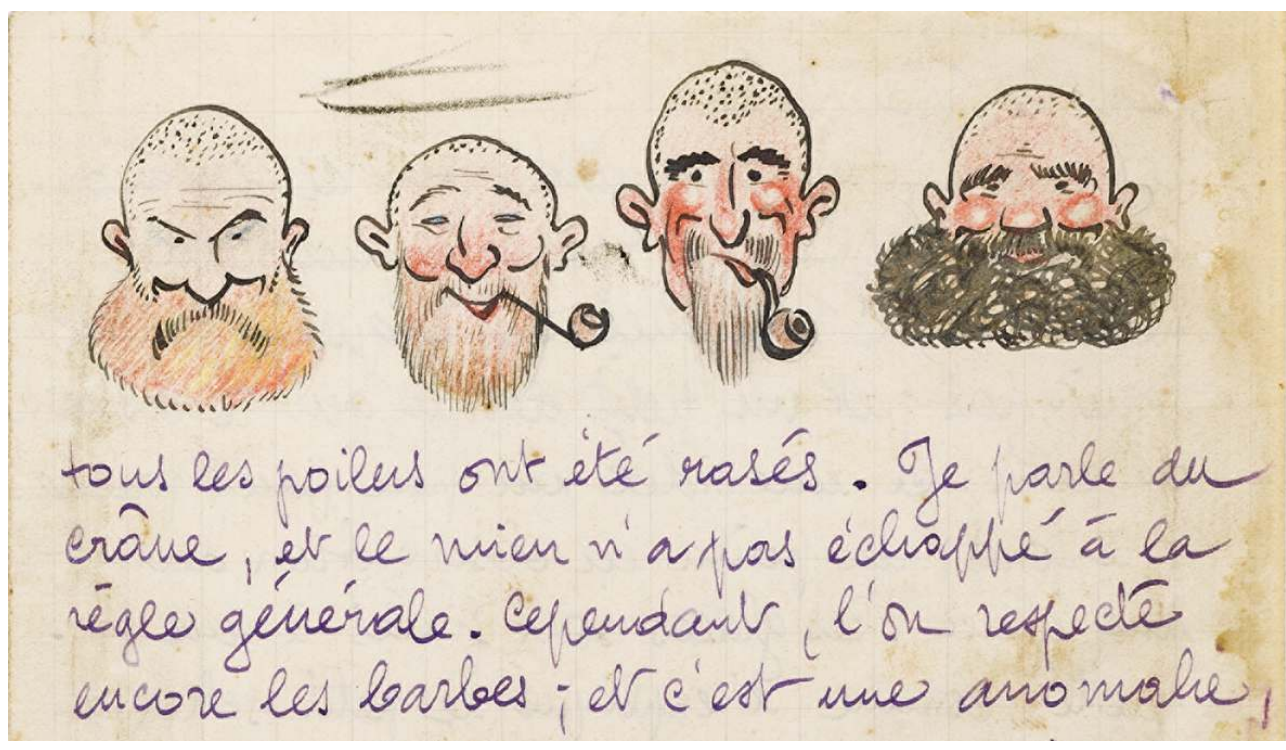

Poilus tondus.

Repro. Barbe-Richaud, Pierre-Marie. @ Droits réservés.

\section{7 avril :}

L'autre jour dans la tranchée, tous les poilus ont été rasés. Je parle du crâne et le mien n'a pas échappé à la règle générale. Cependant, l'on respecte encore les barbes, et c'est une anomalie car elles sont susceptibles de recevoir les mêmes corps étrangers, animés ou non, que le système chevelu avec, en plus, maint déchet de ratatouille périmée (fig. 8). 


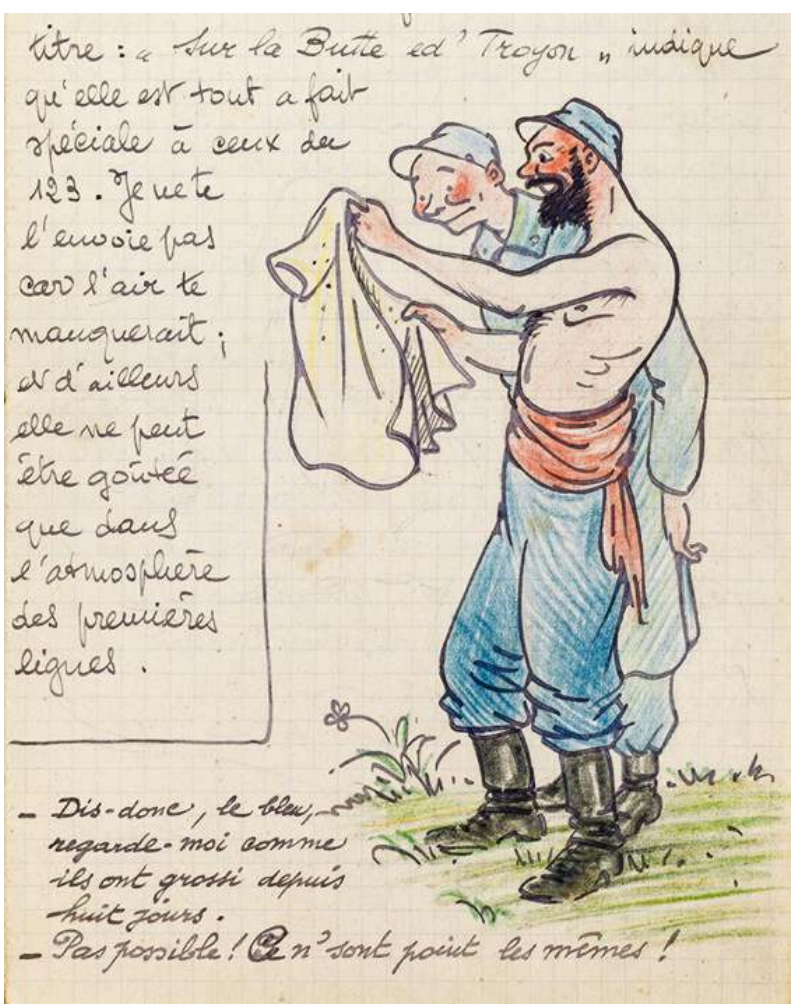

Poilus s'extasiant sur la prospérité des poux.

Repro. Barbe-Richaud, Pierre-Marie. (c) Droits réservés.

Rien de mieux qu'une photo pour rassurer la famille sur sa santé, mais l'obtenir n'est pas chose facile ; 17 avril :

J'espère t'envoyer bientôt une photo qui n'est pas prise dans le cadre que j'aurai souhaité, mais les rares photographes sont tellement pris d'assaut et ont tellement de difficultés à conserver leurs appareils intacts et à se réapprovisionner en plaques et drogues qu'il faut les prendre là où ils sont... je t'envoie une petite photo de la tranchée sur laquelle je ne suis pas.

Troyon-Tranchées, 20 avril :

Mon camarade Demande m'a quitté, hier, et je me trouve un peu seul, étant maintenant l'unique sergent de la section, Il a demandé à être lieutenant et est parti pour suivre pendant une quinzaine un peloton spécial à Merval, en arrière de nos lignes. Je demanderais bien, moi aussi, ne serait-ce que pour me trouver dans le milieu qui me convient; mais je crains de n'avoir pas l'entrain physique nécessaire. Il est vrai que je n'aurai plus l'obligation du sac. J'aurais dû demander en arrivant au dépôt. C'est de ma faute. À l'heure actuelle, il faut une proposition du capitaine, et je crains que ma fatigue récente l'incite à ne pas me proposer. D'ailleurs, si l'on marchait en avant, je boucherais sans doute le premier vide.

Troyon-village, $1^{\text {er }}$ mai 1915 : «Ci-joint ma photo avec un copain. Tu vois que je n’ai pas trop mauvaise mine » (fig. 9). 

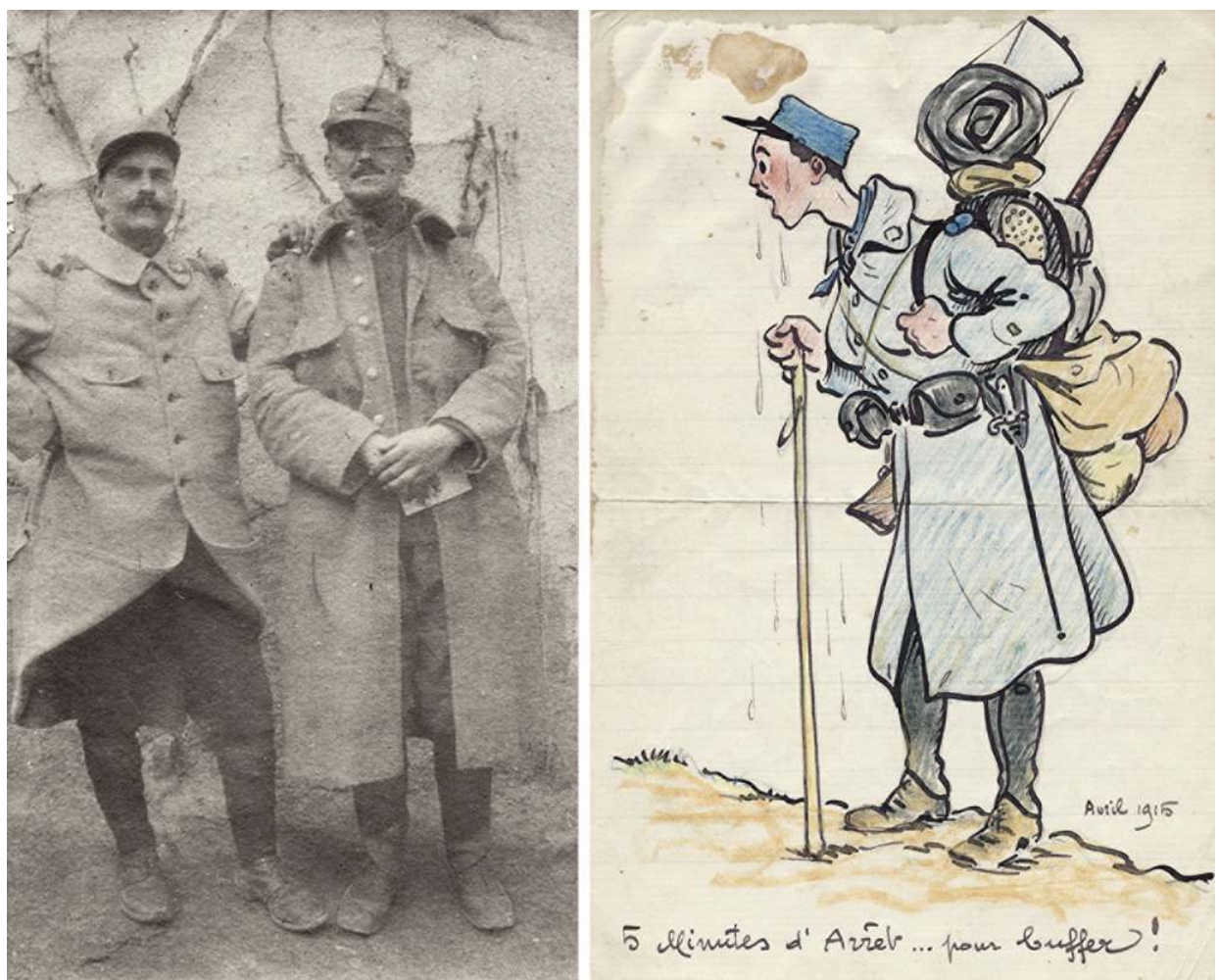

Photo de Pierre Vergez (à gauche) avec un copain et auto caricature.

Repro. Barbe-Richaud, Pierre-Marie. (c) Droits réservés.

Tranchées, $1^{\mathrm{er}}$ juin: «je n'ai pas pu réussir encore à me faire photographier dans la tranchée et pourtant je serai bien content de pouvoir le faire. L'occasion ne s'est pas présentée ».

Le 16 mai :

Trois mois aujourd'hui que j'ai quitté les douceurs du dépôt pour celles du front. Vraiment le temps a passé très vite et je suis tout étonné d'avoir supporté si facilement ce mode de vie que je redoutais à l'avance. Mieux que ça, alors qu'autrefois je toussais des nuits entières à fendre l'âme d'un boche, depuis que je suis ici, je n'ai pas usé une boîte de pastilles et le peu que j'en ai pris, était plutôt par gourmandise.

\section{Caprices de la météo et loisirs des poilus}

La vie s'organise et chacun s'investit pour la rendre plus agréable. Pierre Vergez explique qu'avec le retour du printemps «les tranchées elles-mêmes deviennent plus confortables; et sans aller jusqu'à dire que les poilus auront du regret à les quitter, chacun y a tellement mis du sien qu'il est impossible qu'ils laissent les boches venir s'y installer » (Troyon, $1^{\mathrm{er}}$ avril) (fig. 10). 


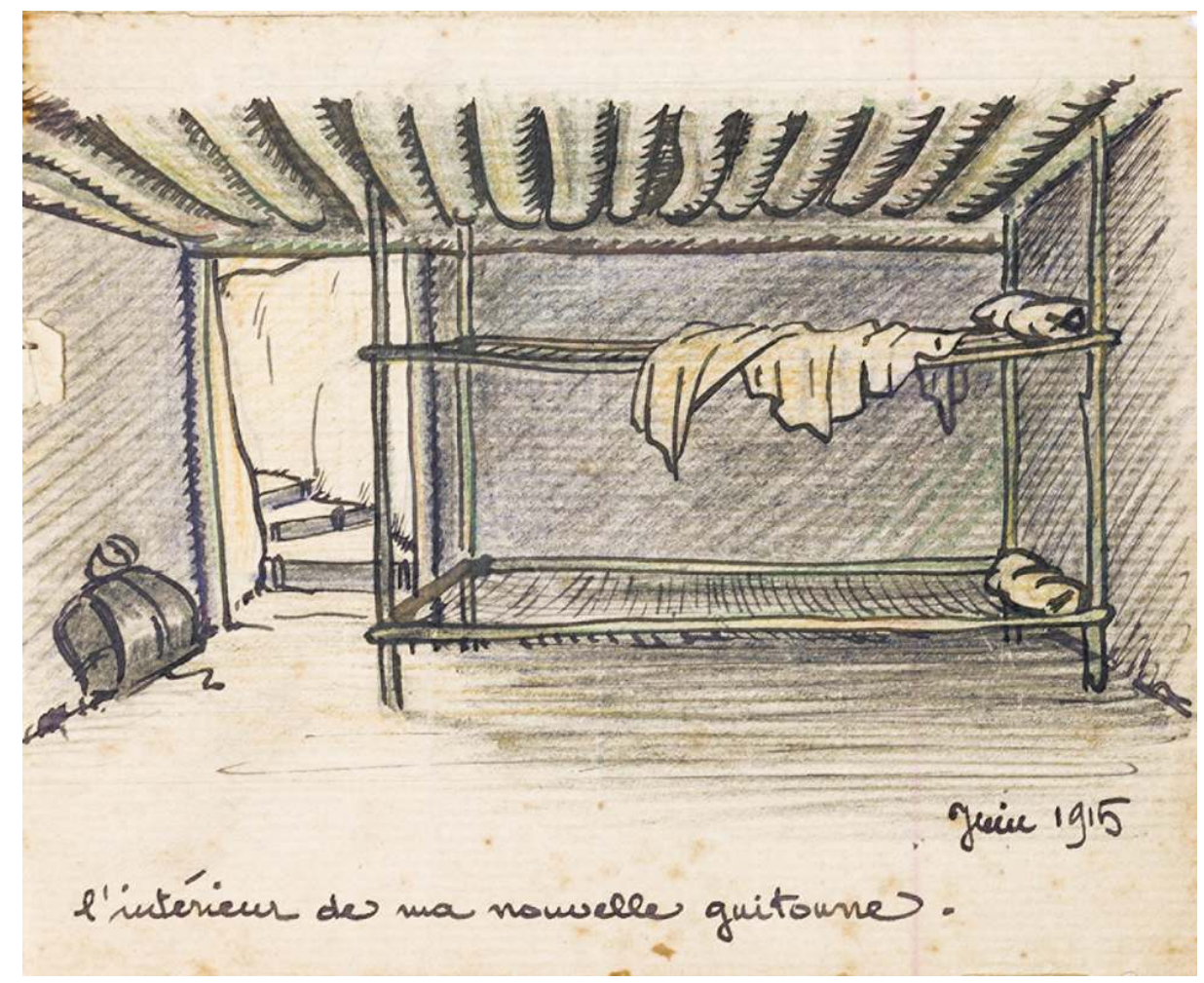

Intérieur d'une guitoune.

Repro. Barbe-Richaud, Pierre-Marie. (c) Droits réservés.

Tranchée, 6 avril : «Vilain temps depuis notre montée. Il pleut constamment, et nous ressemblons à des vers de vase. Je comprends maintenant que l'existence de ces malheureuses bêtes doit être monotone ».

Depuis la tranchée on surveille l'ennemi et, en attendant l'assaut, les temps morts sont nombreux et les hommes s'occupent en jouant aux cartes et en fabriquant de petits objets pour ne pas penser aux jours prochains. Certains confectionnent des bagues d'aluminium faites de débris d'obus allemand ${ }^{7}$. Pierre en envoie une à son père en expliquant en détail la technique de fabrication de l'objet.

Tranchée, 6 avril :

La nouveauté, c'est la bague en aluminium. La tête des obus allemands est faite de ce métal, et il en pousse quotidiennement dans nos campagnes. Aussi chaque poilu a-t-il fait sa récolte. À l'heure actuelle chacun confectionne sa bague d'aluminium. Ce n'est pas une petite affaire. Il faut d'abord débarrasser la tête d'obus des anneaux et vis de cuivre, et de la terre qui l'encombrent ; puis fabriquer un moule en terre et couler dedans le métal en fusion. Il en sort un anneau très grossier qu'il faut ensuite limer très patiemment. C'est l'occupation de tous pendant nos loisirs (fig. 11). 
Figure 11

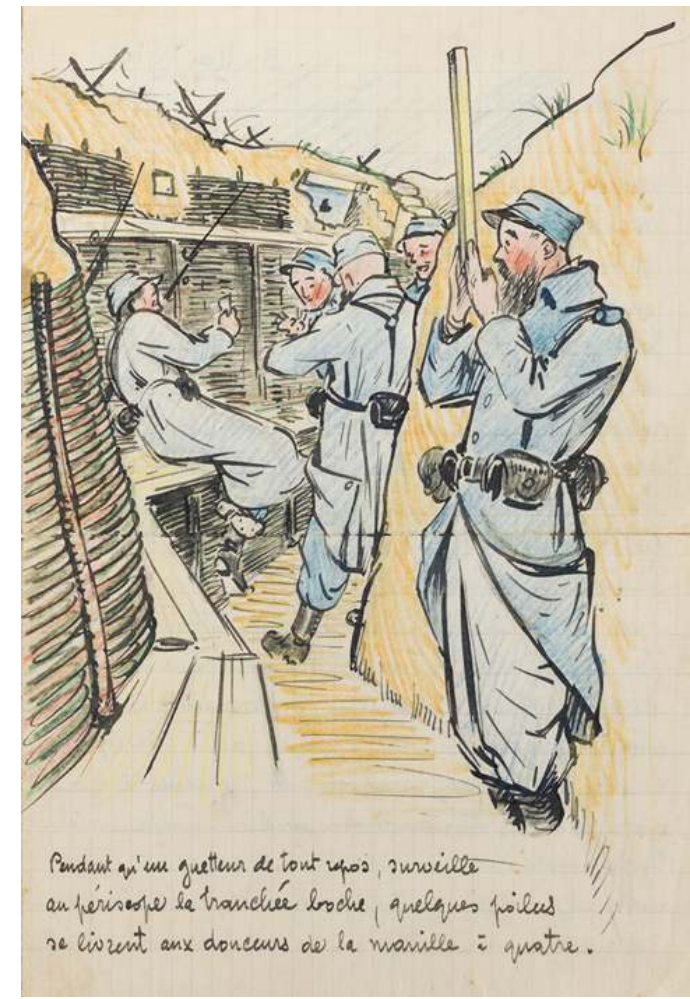

Surveillance et loisirs.

Repro. Barbe-Richaud, Pierre-Marie. (c) Droits réservés.

Moulins, 9 mai :

J'envoie, en même temps que cette lettre un colis contenant: mon plus gros cachenez; quelques balles boches déformées; un couteau fait avec une cartouche anglaise; un bougeoir fabriqué avec deux étuis de fusée. Peut-être le voyage le déformera-t-il un peu. Il aura besoin d'un petit coup d'astiquage, mais il faudra laisser les traces noires qui sont sur les feuilles.

Tranchée, 15 avril : «Le temps a l'air de s'arranger un peu, mais le peu de soleil que nous avons est orageux. Les nuits restent froides et cela gèle un peu au petit jour. Mais tout vaut mieux que la bouillasse dont nous sortons » (fig. 12). 


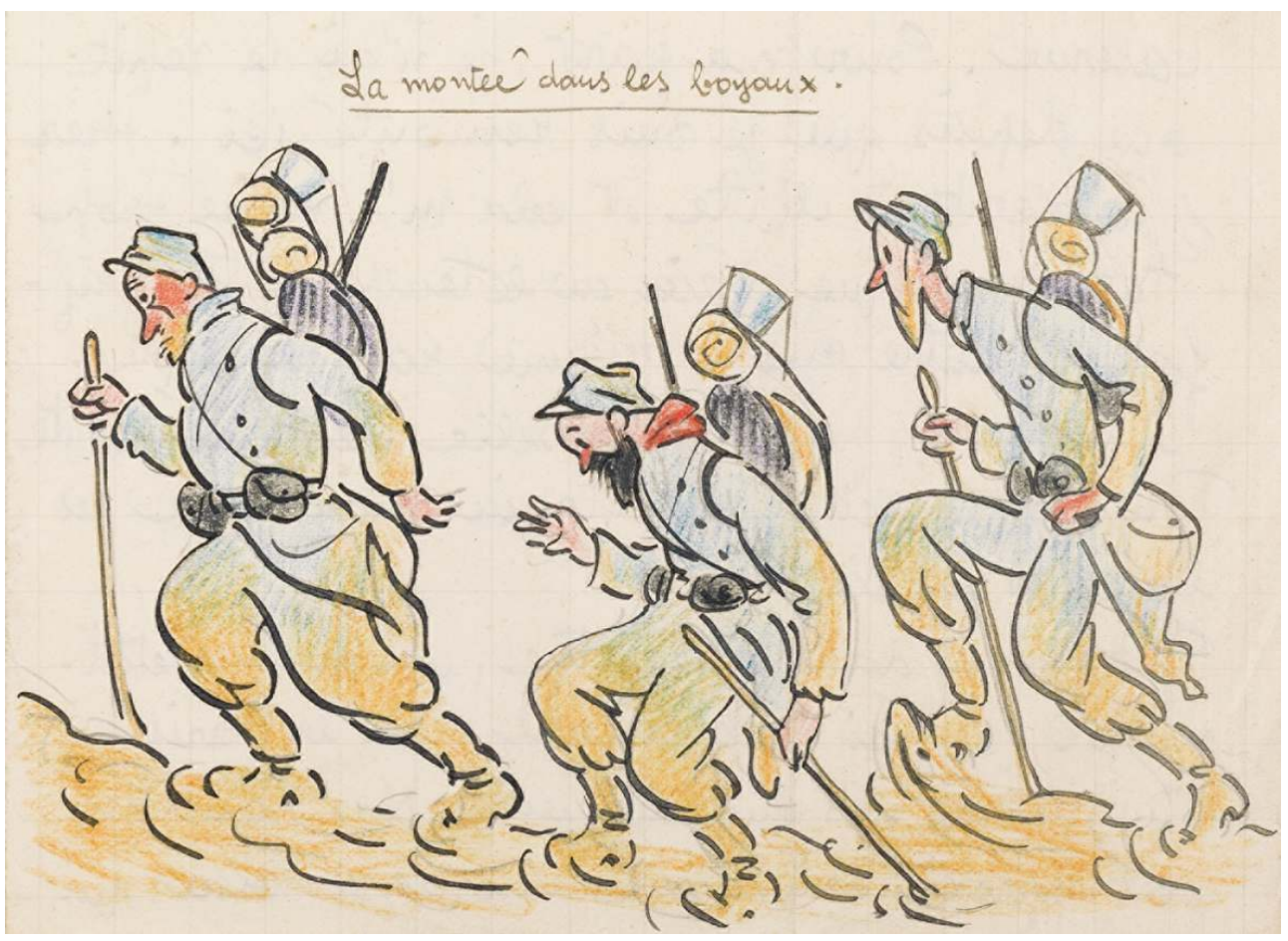

Dans la boue.

Repro. Barbe-Richaud, Pierre-Marie. (c) Droits réservés.

Moulins, 17 avril 1915 : «Comme surcroit d'embêtements voilà que la pluie est revenue, mais il vaut mieux cependant l'avoir ici que pendant notre séjour à la tranchée. Nos quatre derniers jours là-haut ont été ensoleillés, mais d'un soleil d'orage, un peu accablant ».

41 Troyon-village, $1^{\text {er }}$ mai 1915 : « Il fait une chaleur accablante. Heureusement un peu d'air frais se fait sentir, et l'eau ici est abondante. C'est un plaisir d'aller se tremper la tête à la fontaine ».

Troyon-tranchées le 16 mai :

Pour la $1^{\text {ère }}$ fois, il paraît que nous serions relevés dans la journée au lieu de l'être la nuit, comme d'habitude. Cela ne m'étonnerait guère car à partir de demain notre compagnie et la $10^{e}$ qui fait la relève de la nôtre, changent de secteur. Nous allons donc occuper une autre tranchée à 4 ou $500 \mathrm{~m}$. de l'actuelle, vers la gauche, mais nous n'y monterons qu'après nos 4 jours de repos. Ce changement n'est pas pour me déplaire; cela variera un peu les paysages ...Toujours temps orageux le jour, nuits froides avec gelée au petit jour.

Tranchée, 24 mai :

Journée fatigante; un soleil de plomb. Malgré un peu d'air qui souffle, il devient pénible de rester de longues heures éveillé. Pourtant, ayant pris le quart ce matin à 2 heures, il ne faut pas que je songe à dormir avant minuit ce soir, car nous ne serons guère relevés de la tranchée avant 10 ou 11 heures. Pour les hommes, il y a quelque tolérance et je les envoie se reposer par deux ou trois pendant une heure ou deux. Mais pour les gradés, c'est impossible, car les officiers ont leurs abris dans les sapes qui mènent à la première ligne et quelques-uns, dans la tranchée de $2^{\mathrm{e}}$ ligne elle-même... cela fait apprécier rudement les moindres minutes de repos. Aussi, que ce soit sur la paille ou sur les châssis de treillage qui nous servent de lit, 
je te jure que c'est à poings fermés que je dors, sans souci du chahut extérieur ; car l'on finit par s'habituer à la fusillade, aux mitrailleuses, à tout ce qui éclate, comme on se fait peu à peu à Paris au roulement des voitures.

Tranchées, $1^{\mathrm{er}}$ juin :

Ce soir nous quitterons la tranchée vers 9 heures, vraisemblablement pour aller à Moulins, car rien n'a encore été communiqué officiellement au sujet du changement de cantonnement de repos. Le temps continue à être beau, avec des nuits fraîches qui nécessitent un peu d'emmitouflage Le bruit court que le 123 serait appelé à abandonner définitivement son secteur pour une destination que personne, d'ailleurs, ne désigne. Pour ma part, je demeure un peu sceptique, car ce n'est pas la première fois qu'un bruit analogue parcourt les tranchées (fig. 13).

Figure 13
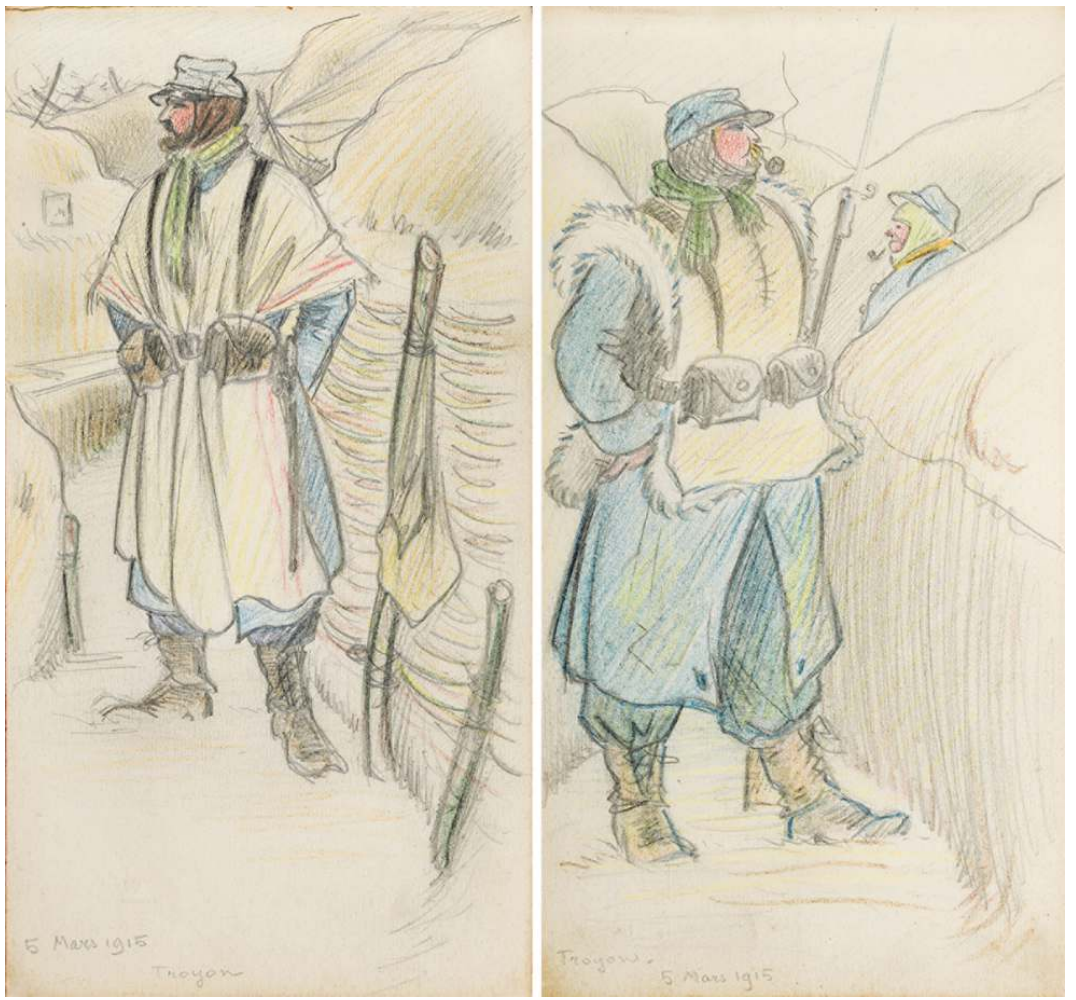

Équipements de fortune contre le froid

Repro. Barbe-Richaud, Pierre-Marie. (c) Droits réservés.

\section{Troyon et Moulins : cantonnements de repos?}

En 1914, Troyon, hameau de la commune de Vendresse, est dépassé par les Allemands dans leur marche vers Paris. Après l'échec de la guerre-éclair, Troyon, déjà en grande partie détruit, se retrouve sur la ligne de front. Pendant la guerre des tranchées ses ruines servent d'abri pour le repos des soldats du front. 
Figure 14

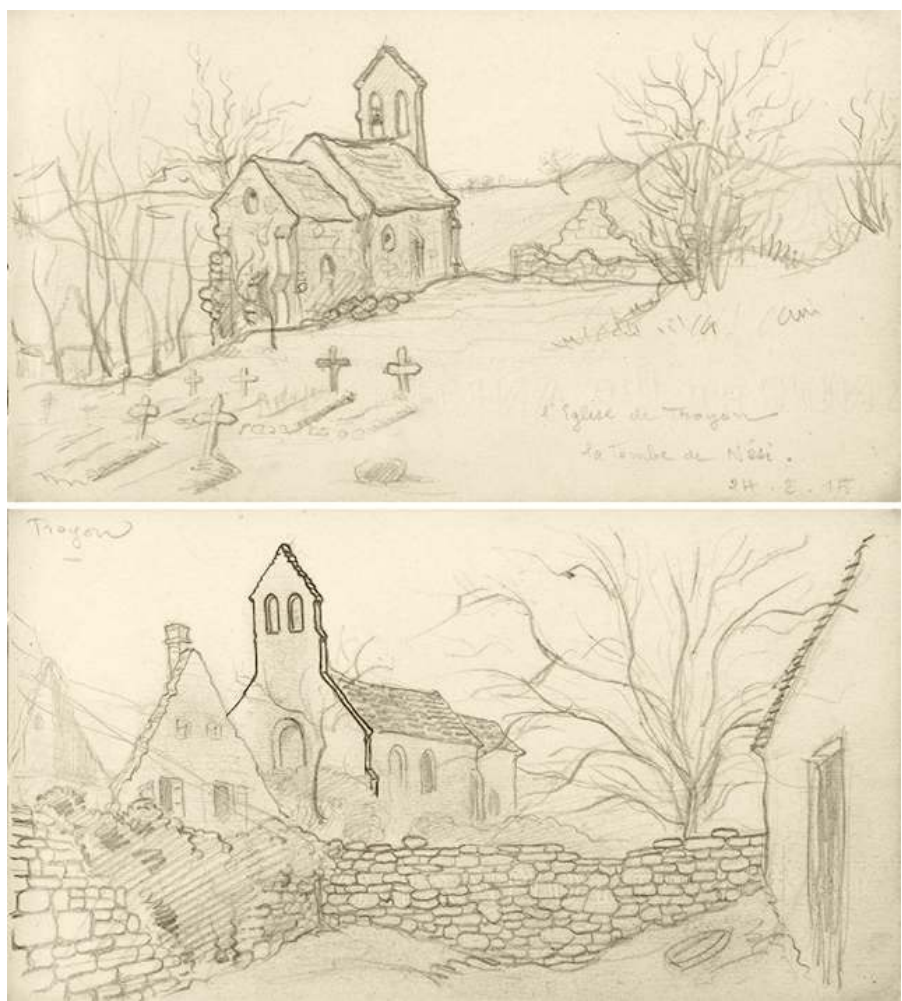

Ruines de Troyon.

Repro. Barbe-Richaud, Pierre-Marie. (c) Droits réservés.

Dans son carnet de croquis Pierre Vergez fait plusieurs dessins au crayon de ce hameau vidé de sa vie : l'un, daté du 24 février 1915, montre le chevet de l'église avec au premier plan des tombes fraîchement creusées, la légende indique le nom d'un des soldats, Nési, probablement un camarade; sur le deuxième, deux murs pignons se dressent face à l'église (fig. 14), les autres représentent une maison debout au milieu des ruines, des vestiges de bâtiments et un petit lavoir isolé. Le plus émouvant est un dessin à l'encre, non daté, dont les couleurs évoquent le drapeau symbole de liberté gagnée au prix du sang : sur une civière près des fonts baptismaux repose le corps d'un soldat couvert du bleu horizon de son manteau, du blanc de son linceul, du rouge du sang qu'il a versé ( fig. 15). 


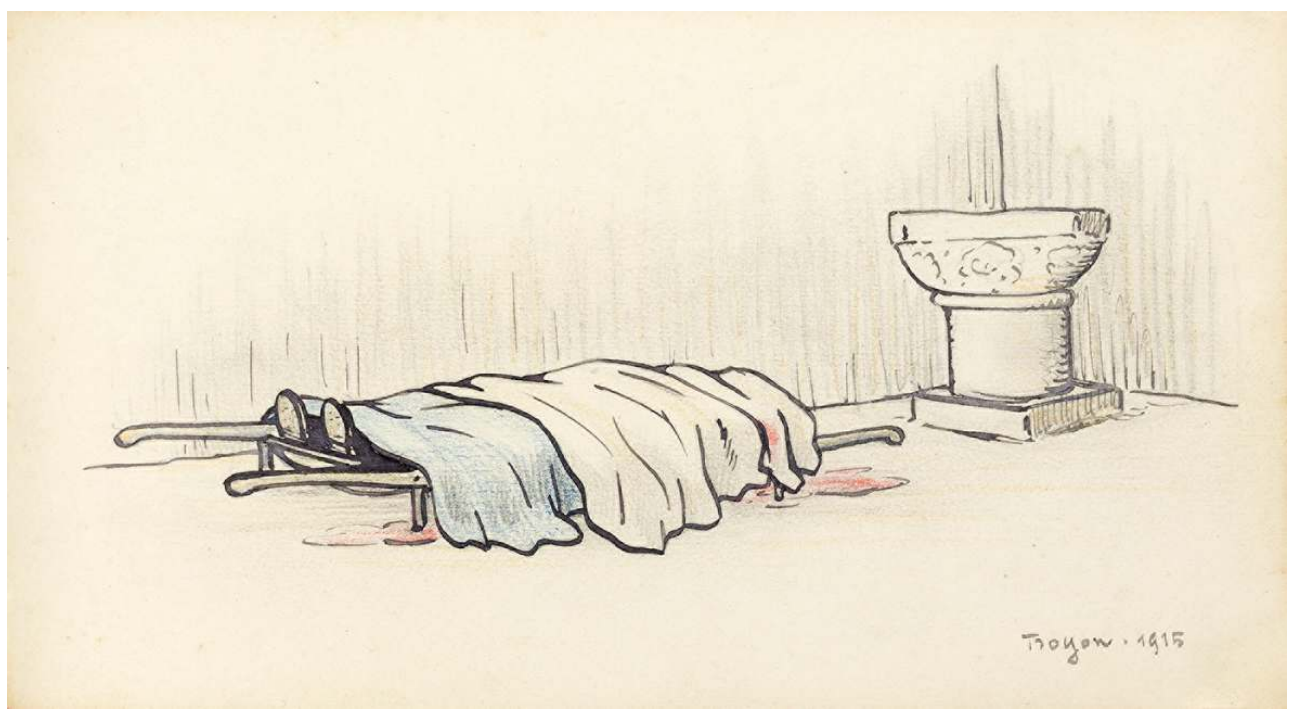

Corps d'un soldat sur une civière déposée près des fonts baptismaux de l'église de Troyon.

Repro. Barbe-Richaud, Pierre-Marie. (c) Droits réservés.

Troyon, $1^{\mathrm{er}}$ avril :

Depuis que le printemps est revenu, un souci de luxe s'affirme partout. À Troyon, où rien n'avait été touché dans le chaos des maisons effondrées, des corvées s'organisent pour mettre quelque ordre parmi les pierres tombées. De vagues trottoirs s'esquissent. Ça et là, grâce au sable roux qui abonde dans la contrée, des allées assez coquettes se dessinent autour de petits massifs qu'ornent des primevères et des fleurs jaunes que je crois être des narcisses. Les tombes de soldats anglais et français qui s'alignent devant la petite église prennent des airs pimpants.

Tranchée, 15 avril :

À notre cantonnement de Moulins nous n'avons pas eu une minute de repos. Aux corvées ordinaires s'ajoutent maintenant des revues, des exercices et des marches. Le but est peut-être excellent, mais tout cela mécontente les hommes qui sont déjà déshabitués de la vie de caserne. Pour ma part, je n'ai de répit que depuis que je suis remonté ici.

50 Moulins, 17 avril : «il est décidément entendu que repos, dorénavant, sera synonyme de surmenage. Il suffit d'être prévenu ».

513 mai :

Hier matin, dimanche, messe dans la petite chapelle de Troyon dont je t'ai jadis envoyé la photo. C'est assez rare ici. À Moulins il y a messe tous les dimanches par l'aumônier militaire, et quelquefois avec partie de chant exécutée par quelques vieillettes qui n'ont pas voulu quitter leurs ruines (fig. 16). 


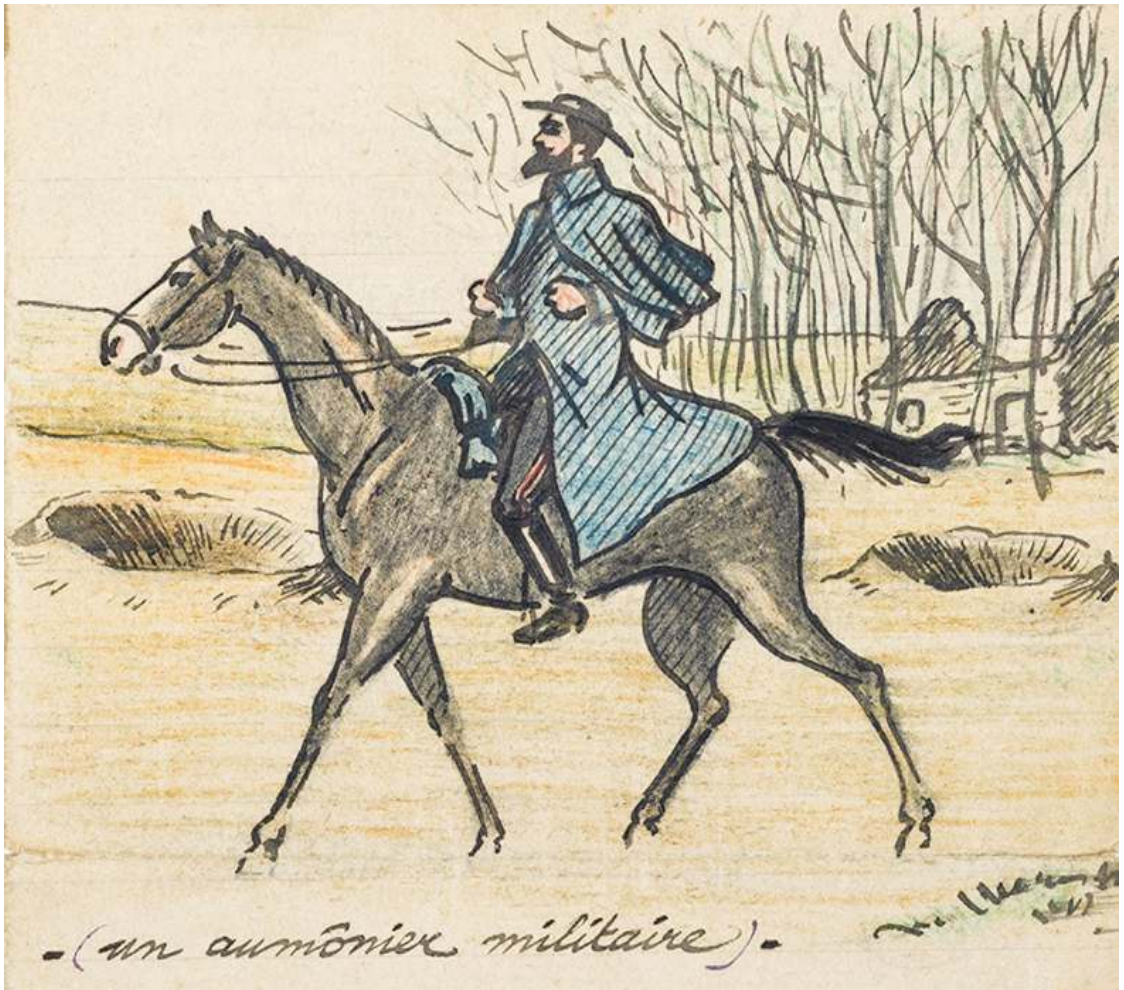

Aumônier militaire sur sa monture.

Repro. Barbe-Richaud, Pierre-Marie. (c) Droits réservés.

Début mai 1915, le village de Moulins est bombardé deux fois. Les mois précédents, Pierre Vergez en avait fait plusieurs dessins montrant ses rues désertes mais l'église comme les maisons y sont encore debout. La vie est encore plus ralentie après, ce que confirme la lettre du 16 mai : «Tu me demandes si j'ai besoin de fonds. Pour le moment pas le moins du monde. Je touche 17 F 20 tous les dix jours, ce qui suffit amplement aux dépenses que nous pouvons faire pendant nos 4 jours à Moulins » (fig. 17). 


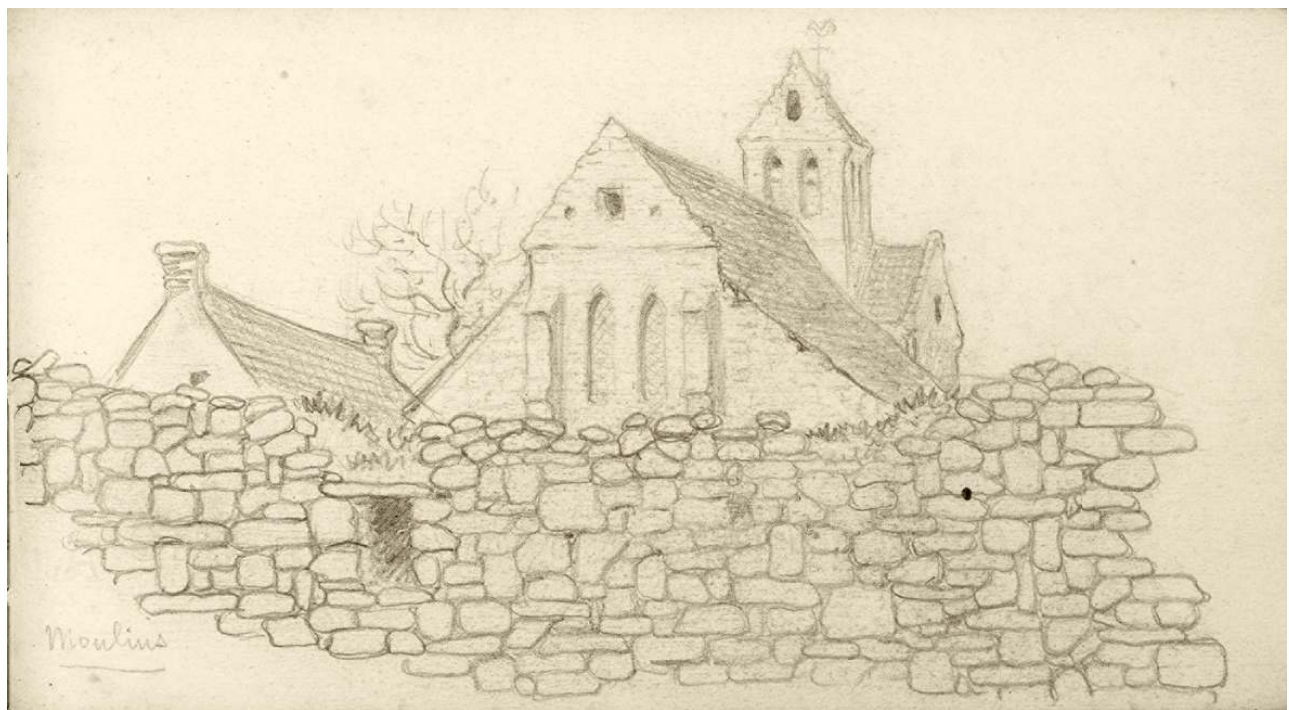

Moulins avant les bombardements de mai 1915

Repro. Barbe-Richaud, Pierre-Marie. (c) Droits réservés.

\section{Tranchées, 24 mai :}

J'ai fait une chanson pour les poilus du $123^{\mathrm{e}}$ et elle se chante déjà dans toute la compagnie et elle est en train de filtrer au dehors. Son titre: "sur la Butte ed' Troyon » indique qu'elle est tout à fait spéciale à ceux du 123. Je ne te l'envoie pas car l'air te manquerait, et d'ailleurs elle ne peut être goûtée que dans l'atmosphère des premières lignes (fig. 18).

\section{Figure 18}

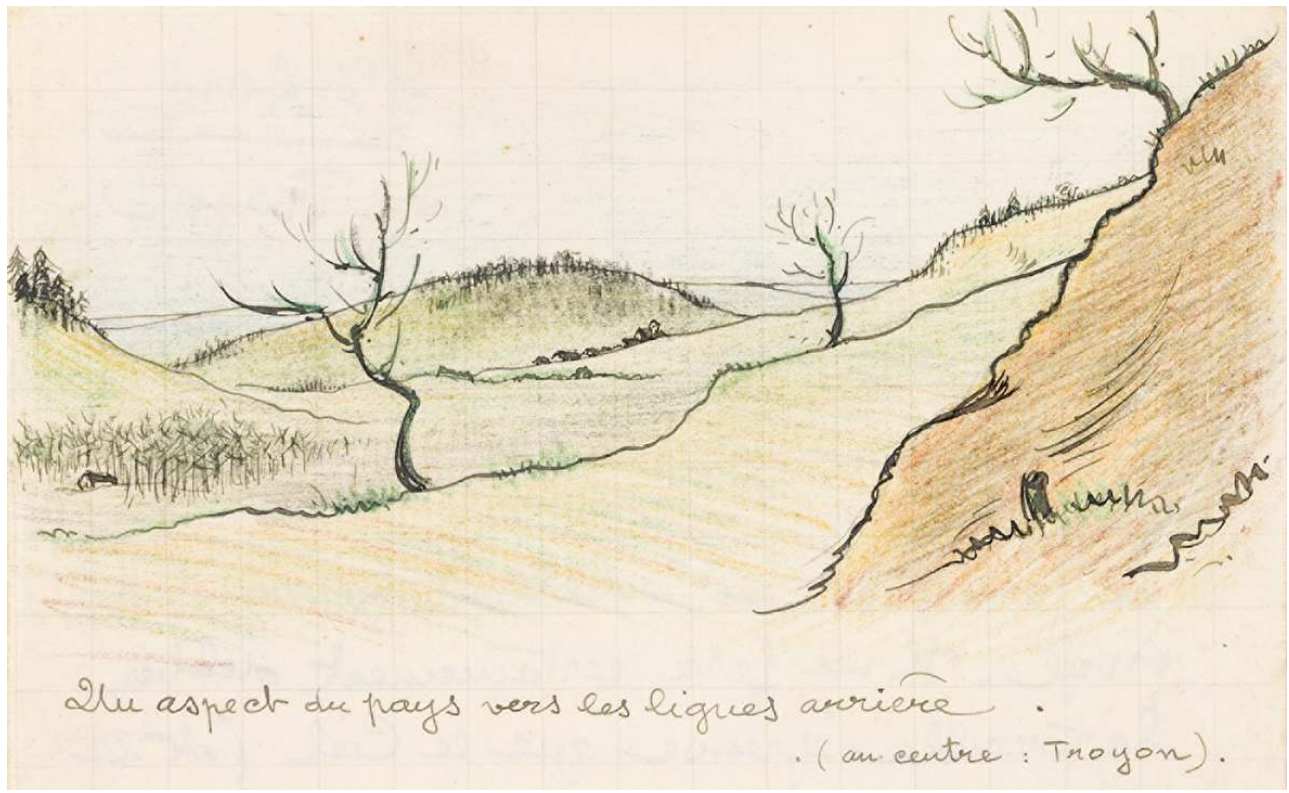

Site de Troyon

Repro. Barbe-Richaud, Pierre-Marie. (c) Droits réservés. 
Tranchées, $1^{\mathrm{er}}$ juin : «Ci-joint 2 photos prises à Moulins récemment avec quelques-uns de mes poilus. Le lendemain, un obus de 105 venait faire une trouée dans le mur qui est derrière nous ».

\section{Courrier et colis : les petits bonheurs du poilu}

Lettre adressée à sa tante le 7 mai 1915 :

L'arrivée du courrier est notre seule joie ici et clôture agréablement les journées les plus mauvaises, car elle a lieu le soir vers huit heures, sauf dans la tranchée, lorsque le temps est trop vilain. Dans ce cas, le facteur attend le petit jour, mais cela arrive rarement. Dans la tranchée, les hommes déchiffrent leur correspondance sous la lune, chacun à sa place, ou, faute de clarté, défilent à tour de rôle dans une guitoune où brûle toujours une veilleuse. Chacun me raconte ses petites histoires et je finis par connaître les femmes de tous mes poilus, leurs avantages et leurs défauts, l'âge des gosses. Je sais que le jeune Émile, qu'on appelle Milou, connaît son alphabet, que Juliette vient de faire sa première communion, et Charlot (celui qui est né depuis la guerre et que son papa ne connait pas encore) ne profite pas beaucoup, rapport que la maman qui le nourrit au sein, se fait trop de bile, et que si la guerre continue, sûr que son lait se mélangera avec ses nerfs... Au cantonnement, la lecture des lettres se fait en groupe, à la lueur des rares bougies; elle est suivie d'interminables commentaires. Ceux qui n'en reçoivent presque jamais s'approchent des groupes des privilégiés et, mélancoliquement, hument les bouffées d'intimité et les effluves d'affection qui se dégagent des enveloppes reçues par les voisins plus heureux.

Le 7 juin, il annonce à son père la censure du courrier :

Une note a passé récemment, renouvelant l'interdiction de mentionner aucun nom de localité dans la correspondance. Aussi pour éviter tout ennui ou tout retard, je me contenterai d'initiales tant que nous resterons dans notre secteur habituel. D'après la note en question, l'inspection de la correspondance se fait en bloc, c'està-dire qu'à telle ou telle date, toutes les lettres émanant de telle ou telle unité sont visitées. Ne t'étonne donc pas si quelque lettre subit un assez long retard. Sans doute quelque indiscrétion grave a-t-elle été découverte par hasard d'où cette crise inquisitoriale.

Dans sa lettre du 20 avril, il écrit :

Je t'accuse réception du petit colis reçu ce matin, Merci une fois de plus, Les journaux du 15 et 16 sont venus en même temps... (fig. 19) De plus, tu peux t'abstenir maintenant de m'envoyer papier et enveloppes jusqu'aux premiers jours de mai. J'ai fait l'inventaire de mes poches, et j'y ai trouvé de quoi écrire une vingtaine de lettres. Je suis donc suffisamment riche. À force de bourrer mes poches de poitrine de carnets, album et papiers divers, je finis par ne plus pouvoir boutonner ma capote et arborer des seins indécents. Mon camarade Demande, lui, porte ses paperasses sur le ventre, et Dieu sait qu'il les collectionne. Cela nous donne des allures assez intéressantes ainsi qu'essaie de l'indiquer le mauvais croquis ci-joint, Donc trêve de papier pendant une quinzaine environ. 
Figure 19

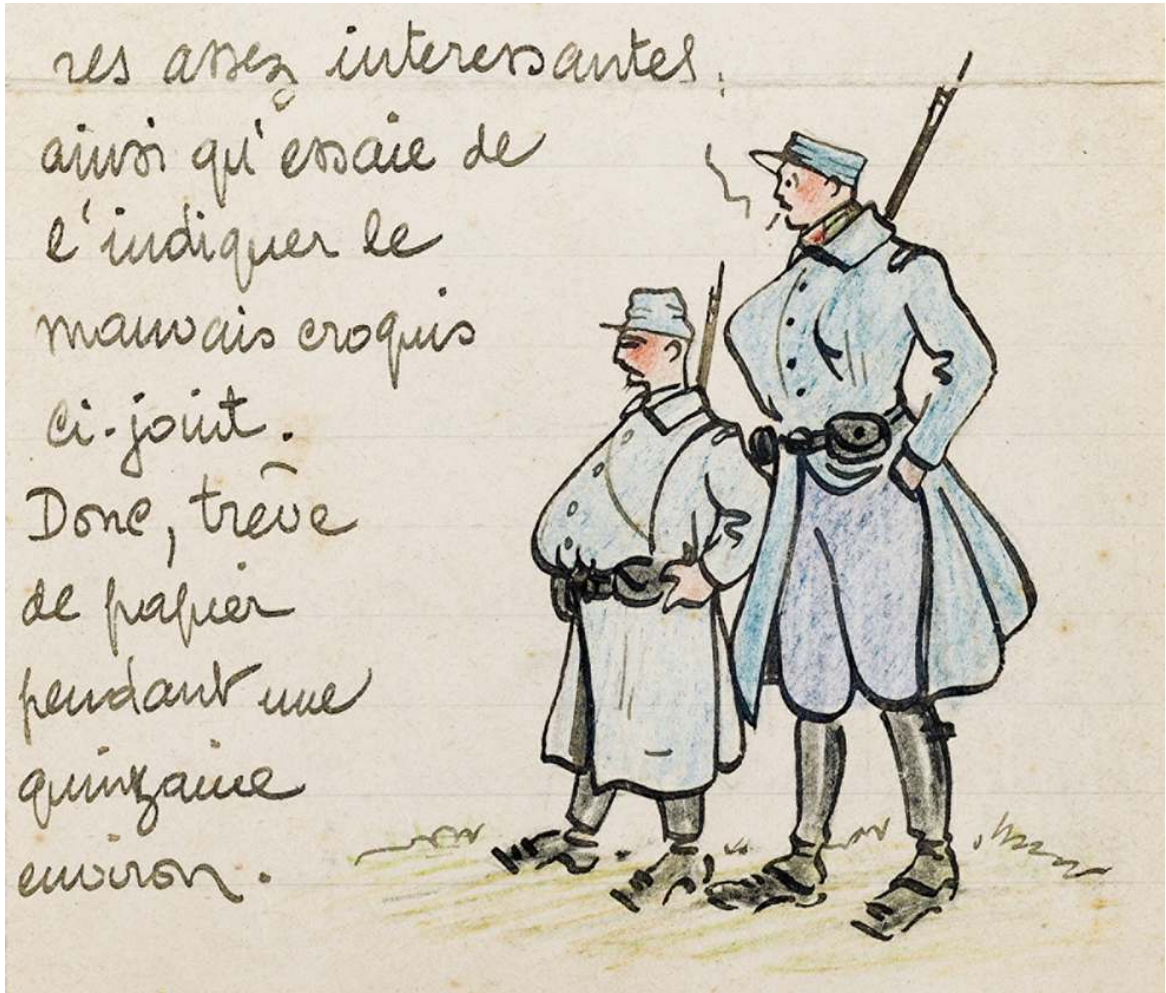

Pierre Vergez et son camarade Demande, les poches bourrées de leurs lettres et papiers personnels. Repro. Barbe-Richaud, Pierre-Marie. (c) Droits réservés.

L'ordinaire n'est ni copieux ni très appétissant et l'améliorer est une des grandes préoccupations du poilu, aussi les victuailles prennent-elles une place importante dans le contenu des colis (fig. 20). 

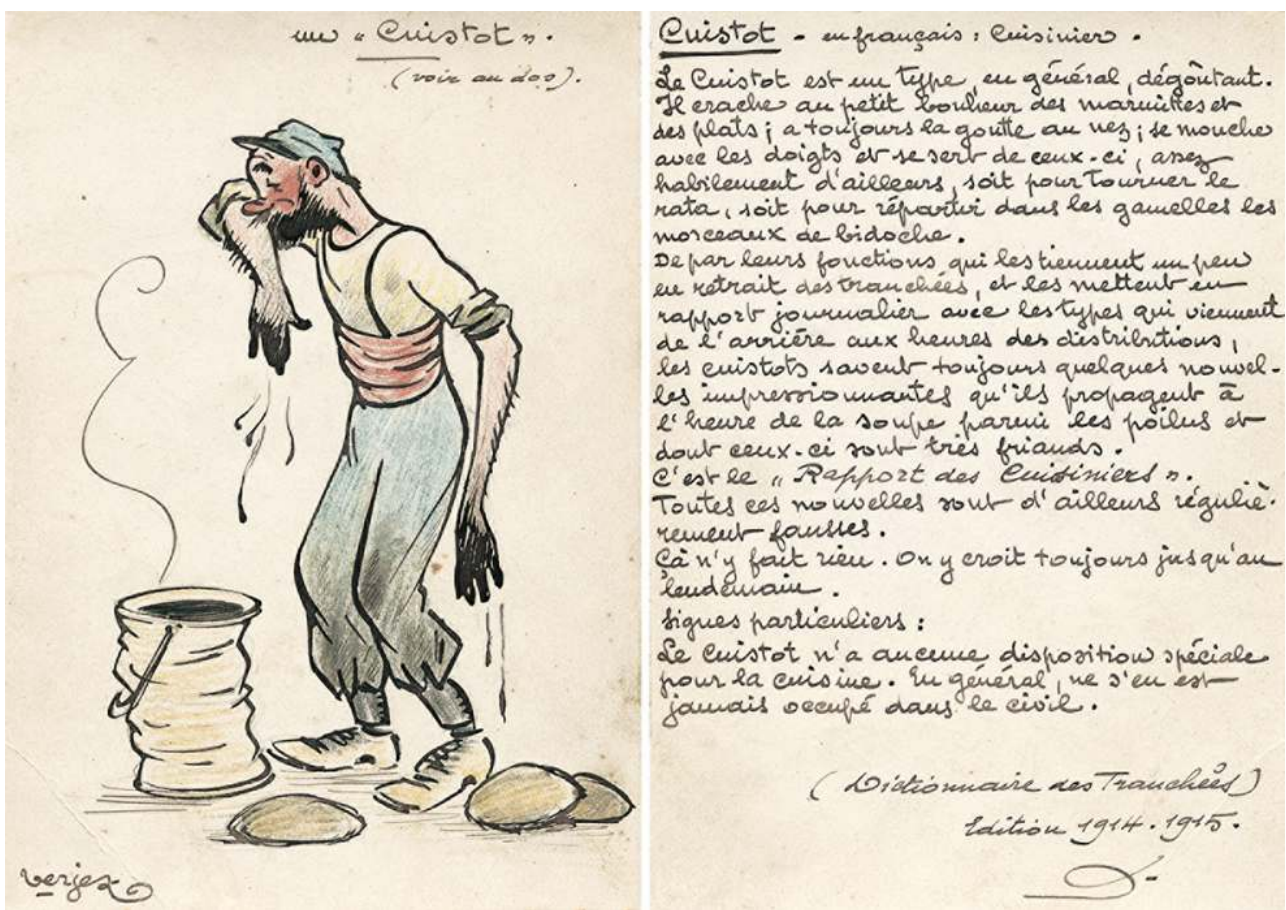

Définition illustrée du cuistot.

Repro. Barbe-Richaud, Pierre-Marie. (c) Droits réservés.

\section{Tranchée, 29 mars 1915 :}

Tu pouvais me donner en mille le contenu de ton colis, en revanche, tu ne peux pas te faire l'idée la plus vague de l'état dans lequel il est arrivé. Je pense t'en donner un aperçu en te disant que le poisson de chocolat semblait avoir retrouvé son élément et souffrir atrocement de ne pouvoir agiter ses nageoires. Cependant, Dieu sait par quel miracle, un œuf, un seul, avait échappé au cataclysme dont la visqueuse horreur avait filtré hors de la boîte métallique, baignant exactement les tablettes de chocolat et transformant le papier à lettres en une sorte de papier à mouches du plus curieux aspect... Enfin nous avons bien ri dans la guitoune, et plusieurs camarades, pour me consoler, ont affirmé que tout cela était fait exprès, et que c'était là justement que résidait le poisson d'avril.

Tranchée, 3 avril :

Le poulet a passé un vilain quart d'heure jeudi soir. Mon camarade Demande et moi, nous rappelant à temps que le lendemain était vendredi saint, la liquidation du malheureux bipède a été décidée, puis consommée. Le vendredi, notre maigre a consisté : le matin, en une purée de pommes de terre somptueuse et une boîte de sardines chacun; le soir, en une assiettée de haricots (plein bords), plus deux camemberts à nous deux. Tu vois que ce jeûne ne nous aura pas trop éprouvés. Cependant, pour nous récompenser de notre attitude héroïque devant la tentation des viandes de l'ordinaire, nous nous sommes permis tous les deux d'escamoter ce matin un pâté de foie familial reçu par le camarade en question. Des tartines de beurre (du tien) trempées dans le quart de jus réglementaire ont constitué un dessert succulent et maintenant, nous pouvons attendre sans impatience la ratatouille de 5 heures.

61 Le 12 avril, Pierre demande à son père de faire en sorte que ses colis lui parviennent quand il est dans la tranchée: «c'est là-haut surtout que se fait sentir la monotonie de 
l'ordinaire. Tu peux appuyer sur le chocolat à volonté. De plus je me rappelle un petit rôti de veau piqué à l'ail dont j'aurais plaisir à avoir une copie ».

Sur une lettre datée du 17 avril, Pierre dessine « un poilu délicat soufflant sur son quart plein de café, de façon à chasser la couche superficielle qui entraine avec elle la kyrielle des corpuscules variés qui surnagent » (fig. 21).

Figure 21

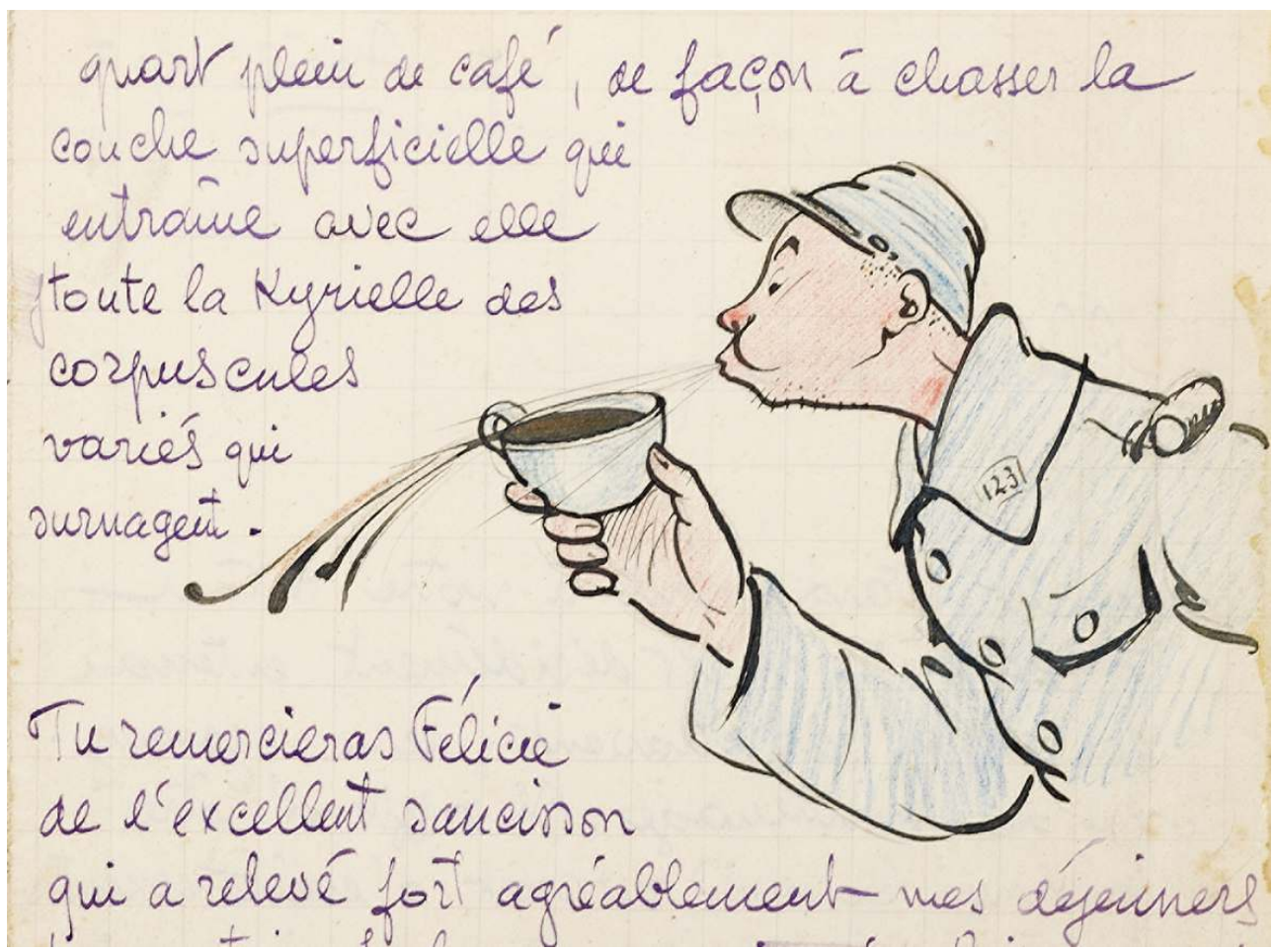

Poilu soufflant sur son quart de café.

Repro. Barbe-Richaud, Pierre-Marie. (c) Droits réservés.

Troyon-tranchée, 20 avril : «Je recevrais avec plaisir des œufs durs, si tes poules ne sont pas en grève ».

Troyon-village, $1^{\mathrm{er}}$ mai 1915 : «J'ai reçu ce matin le colis : œufs durs et bananes. J'ai fort apprécié les uns et les autres et te sais gré d'avoir pensé même à l'assaisonnement. Dorénavant, choisis des bananes simplement jaunes, car les noires sont très mures et arrivent un peu liquides ».

3 mai :

J'ai reçu aujourd'hui d'amis de Paris une petite fiole de menthe Ricqlès et de l'eau de Cologne... il y a eu de la perte sur ton dernier colis : j'ai dû jeter, avec quels regrets, quatre œufs qui sentaient vraiment mauvais. D'où cela vient-il ? Peut-être de la chaleur. En tout cas il y a quelque chose, car étant cueillis à la poule même, ils ont dû quitter Bouho-Bén en parfait état.Je pense qu'il vaut mieux que tu t'abstiennes de beurre car il commence à arriver aux camarades un peu fondu.

Lors du deuxième bombardement de Moulins, début mai, plusieurs écuries ont été touchées et outre les chevaux de troupe,

quelques canassons civils ont écopé aussi, et nous allons nous en ressentir, car ils appartenaient aux rares commerçants qui soient restés ici, et servaient à ceux-ci pour conduire les vieilles guimbardes qui allaient chercher à Fismes les provisions. 
Il doit cependant en rester quelques-uns et nous pouvons espérer encore pouvoir nous procurer les bouteilles de vin, camemberts, ananas et abricots de conserve, qui constituent le comble de notre luxe... Le petit pâté et le jambon d'York qui calaient la flanelle étaient délicieux, vraiment.

$671^{\mathrm{er}}$ juin :

Je sais très bien qu'il est impossible de sortir des conserves de charcuterie et de poisson. C'est très bien ainsi ; et il en est beaucoup qui doivent se contenter des ratas de l'ordinaire qui manquent franchement de variété. Pour les boîtes de légumes, il vaut mieux que tu t'abstiennes. Il faut avoir recours aux cuisiniers pour la cuisson, et il est difficile de ne pas leur en offrir ce qui restreint considérablement les rations. Tiens-toi donc aux sardines, thon et charcuteries en boîte. Mon camarade Demande, revenu du peloton, partage avec moi ses colis et je partage avec lui les miens, sauf le chocolat et le tabac. Ce soir, nous portons un coup fatal à une poche de figues et raisins qu'il ne veut pas retourner à Moulins.

7 juin : «J'ai reçu le petit pot de confiture. Le contenu était excellent. Si tu m'en renvoies, ne change pas de fruits. C'était des prunes d'Agen en pâte dense analogue au raisiné. Je ne pense pas qu'il y ait de pâte plus solide. Malgré cela, elle avait un peu coulé à l'extérieur ».

\section{Le goût de la nature : des pois de senteur dans les tranchées}

Au milieu de ces paysages massacrés, torturés, l'amour de la nature, parfois teinté de nostalgie, est encore plus vivace. Faire entrer un peu de beauté dans les tranchées semble une aberration, et pourtant...

Moulins, 9 mai :

La campagne a reverdi sérieusement depuis notre dernier séjour. Dans le jardin qui attient à notre cantonnement il $\mathrm{y}$ a surtout des groseilliers dont les petites grappes sont déjà formées. Cela m'a rappelé Flers où nous en avions beaucoup. On les appelait des «grades». Tout cela est bien loin... si cela n'est pas devenu rare, tu devrais bien m'envoyer quelques graines de capucines, volubilis et pois de senteur. J'aurais plaisir à en semer aux abords de ma guitoune, dans la tranchée, où je pense avoir le temps de les voir fleurir. Je parle sérieusement.

71 Tranchées, 24 mai :

Les graines sont semées depuis trois jours. Un de mes hommes, jardinier, a arrangé cela pour le mieux et a semé de la graine de radis qui nous promet quelques hors d'œuvre. Je vais passer ce soir la consigne à mon remplaçant de guitoune (un sergent de la $10^{\mathrm{e}} \mathrm{C}^{\mathrm{ie}}$ ) pour l'arrosage. Il me tarde de remonter dans 4 jours, de Troyon, pour voir les premières pousses.

Tranchées, $1^{\mathrm{er}}$ juin :

Mes fleurs poussent. Tout est sorti. Les haricots battent les records et atteignent déjà 5 à 6 centimètres. Les radis plus modestes sur plusieurs rangs dans un ordre parfait. S'il est un peu présomptueux de penser voir fleurir les liserons, j'espère au moins pouvoir profiter des radis (fig. 22). 


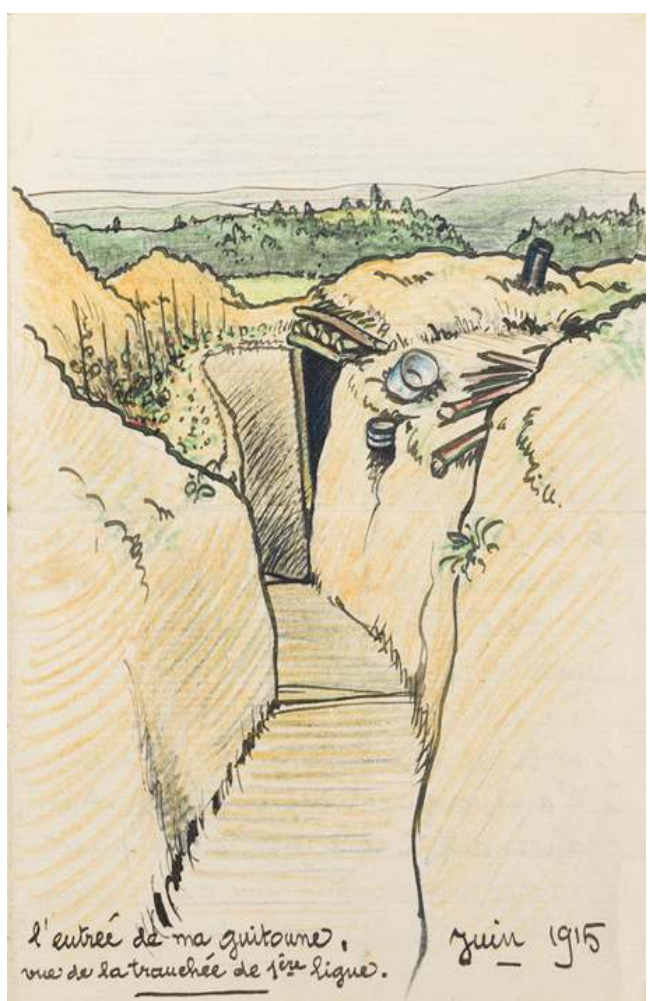

Plantation de fleurs et légumes en face de la guitoune.

Repro. Barbe-Richaud, Pierre-Marie. (c) Droits réservés.

\section{Dernière lettre, T., 11 juin 1915 :}

Je t'écris ce matin dans le cadre le plus rustique qui soit. Je remplis ce matin de $6 \mathrm{~h}$ à 11 heures les fonctions de garde forestier dans les bois de $\mathrm{T}$. où nous prenons comme à l'ordinaire notre repos. Ces fonctions consistent à empêcher que quiconque abatte de nouveaux arbres. Depuis des mois que des soldats cantonnent par ici, ces malheureux bois ont été bien éprouvés par la hache des cuisiniers et éclaircis par tous les prélèvements qui ont été nécessaires à l'aménagement des tranchées. Aussi, maintenant il reste juste suffisamment d'arbres pour abriter aux vues les mouvements de troupes; et l'ordre est venu de ne plus faire de nouvelles victimes. À tour de rôle, les sergents sont désignés pour veiller à ce que l'ordre ne soit pas enfreint. Par beau temps c'est une corvée très agréable, le pays étant fort plaisant à l'œil. Avec un peu moins de brusquerie dans les pentes, il rappelle les abords de Bagnères du côté du camp de César, collines et vallons, avec alternances de prairies et de futaies. Par endroits, malgré la proximité des boches, il y a même des terrains cultivés, de fort jolis champs de blé ou de pommes de terre, œuvre des artilleurs. Mais c'est l'exception. Presque tout le terrain est inculte et les coquelicots s'en donnent à rouge que veux-tu. Sur les pentes des collines ou au creux des vallées, ils figurent de larges flaques ou de longues trainées sanglantes où semble s'épanouir en une vie nouvelle tout le rouge qui a déjà coulé par là. 


\section{NOTES}

1. - Allusion à une rumeur disant qu'il y aurait pénurie de nourriture en Allemagne: voir KIPLING, Rudyard. Tu seras un homme mon fils, suivi de Lettres à son fils. Paris : Éditions Mille et une nuits, 1998, p. 51.

2. - Parmi les chansons dont Pierre Vergez signe l'illustration sous le nom de « Verjez », on peut citer celles du compositeur parolier F. L. Benech (Maryvonne, C'est pour ça qu'on les aime, Petite poupée jolie, La Jolie Loucheuse) et des compositions de Léo Daniderff (Le grand frisé, chanson réaliste, Berceuse tendre, La noce à Ferdinand, Quand je vois Mimile «chanson excentrique »; il dessine encore la page de titre de Tout's les femmes (1910) et Le chapeau à la main (1911) créées par Félix Mayol, chanteur vedette du music-hall et un portrait du chansonnier Georgius pour Elle était vraiment charmante. Il donne aussi des planches à la revue satirique Le Sourire.

3. - En décembre 1918 il a droit à une permission qu'il passe chez son père, mais il doit rejoindre son régiment en Alsace pour être démobilisé. C'est là qu'il va attraper la « grippe espagnole » qui a fait plus de 400000 victimes en France.

4. - Historique du $123^{e}$ Régiment d'Infanterie pendant la campagne 1914-1919. Paris : Librairie Chapelot, 1919.

5. - KIPLING, Rudyard. Tu seras un homme mon fils, suivi de Lettres à son fils. Paris : Éditions Mille et une nuits, 1998.

6. - KIPLING, Rudyard. Tu seras un homme mon fils, suivi de Lettres à son fils. Paris : Éditions Mille et une nuits, 1998, p. 47, 52, 63 et 65.

7. - Côté anglais on s'adonnait aux mêmes activités : le 30 août 1915, John Kipling dit à son père qu'environ un homme sur quatre porte une bague d'aluminium faite de débris d'obus allemand ( op. cit., p. 69).

\section{ABSTRACTS}

Pierre Vergez was a poster designer and cartoonist whose career in Paris was only just beginning when war broke out in 1914. He was mobilised with the $123^{\text {rd }}$ infantry regiment of La Rochelle which was sent to the front, where he wrote regularly to his father. Amongst these letters are the illustrated ones that he sent from the trenches or from his quarters at Troyon and Moulins. Although he was immersed in all the horrors of the war, Vergez kept his sense of humour and his capacities of observation which are visible in his texts accompanied by sketches and caricatures done in ink, in pencil or in colouring crayons.

Pierre Vergez, affichiste-humoriste, commence à faire carrière à Paris lorsqu'éclate la guerre de 1914-1918. Aussitôt mobilisé dans le $123^{\mathrm{e}}$ régiment d'infanterie de La Rochelle, il part sur le front d'où il écrit régulièrement à son père. De cette correspondance subsistent des lettres illustrées envoyées des tranchées ou des cantonnements de Troyon et Moulins. Bien que plongé dans l'horreur de la guerre, il garde un sens de l'humour et de l'observation qu'il manifeste dans ses écrits rehaussés de dessins et de caricatures à l'encre, à la mine ou aux crayons de couleur. 
INDEX

Mots-clés: Vergez, Verjez, tranchée, poilu, Troyon, Moulins

Keywords: trench, 'poilu'

\section{AUTHOR}

\section{BRIGITTE FROMAGET}

chercheur au Service Patrimoine et Inventaire brigitte.fromaget@sfr.fr 